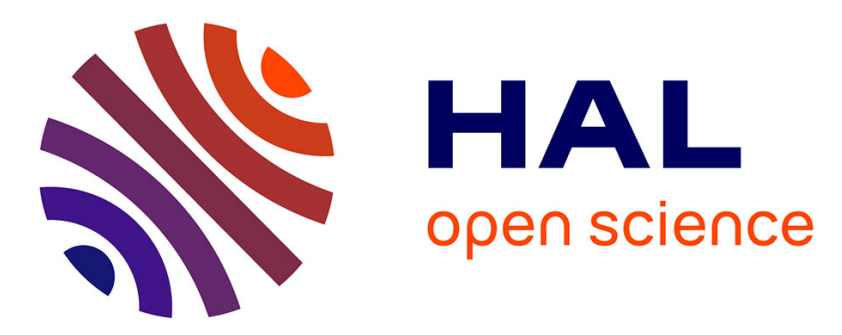

\title{
Investigation and modeling of the effects of light spectrum and incident angle on the growth of Chlorella vulgaris in photobioreactors
}

\author{
Antoine Soulies, Jack Legrand, Helene Marec, Jeremy Pruvost, Cathy \\ Castelain, Teodor Burghelea, Jean-François Cornet
}

\section{To cite this version:}

Antoine Soulies, Jack Legrand, Helene Marec, Jeremy Pruvost, Cathy Castelain, et al.. Investigation and modeling of the effects of light spectrum and incident angle on the growth of Chlorella vulgaris in photobioreactors. Biotechnology Progress, 2016, 32 (2), pp.247-261. 10.1002/btpr.2244. hal01950701

\author{
HAL Id: hal-01950701 \\ https://hal.science/hal-01950701
}

Submitted on 14 Apr 2020

HAL is a multi-disciplinary open access archive for the deposit and dissemination of scientific research documents, whether they are published or not. The documents may come from teaching and research institutions in France or abroad, or from public or private research centers.
L'archive ouverte pluridisciplinaire HAL, est destinée au dépôt et à la diffusion de documents scientifiques de niveau recherche, publiés ou non, émanant des établissements d'enseignement et de recherche français ou étrangers, des laboratoires publics ou privés. 


\title{
Investigation and Modeling of the Effects of Light Spectrum and Incident Angle on the Growth of Chlorella vulgaris in Photobioreactors
}

\author{
Antoine Souliès, Jack Legrand, Hélène Marec, and Jérémy Pruvost \\ CNRS, Laboratoire de Génie des Procédés - Environnement - Agroalimentaire, Université De Nantes, UMR 614437 Boulevard De \\ L'université, BP 406, Saint-Nazaire Cedex, 44602, France
}

\section{Cathy Castelain and Teodor Burghelea}

CNRS, Laboratoire de Thermocinétique de Nantes, Université De Nantes, UMR 6607, La Chantrerie, Rue Christian-Pauc, BP 50609, Nantes Cedex 3, 44306, France

\section{Jean-François Cornet}

Université Clermont Auvergne, Sigma-Clermont, Institut Pascal, UMR CNRS 6602, BP 10448, F63000 Clermont-Ferrand, France

\begin{abstract}
An in-depth investigation of how various illumination conditions influence microalgal growth in photobioreactors $(P B R)$ has been presented. Effects of both the light emission spectrum (white and red) and the light incident angle $\left(0^{\circ}\right.$ and $60^{\circ}$ ) on the PBR surface were investigated. The experiments were conducted in two fully controlled lab-scale PBRs, a torus PBR and a thin flat-panel PBR for high cell density culture. The results obtained in the torus $P B R$ were used to build the kinetic growth model of Chlorella vulgaris taken as a model species. The PBR model was then applied to the thin flat-panel PBR, which was run with various illumination conditions. Its detailed representation of local rate of photon absorption under various conditions (spectral calculation of light attenuation, incident angle influence) enabled the model to take into account all the tested conditions with no further adjustment. This allowed a detailed investigation of the coupling between radiation field and photosynthetic growth. Effects of all the radiation conditions together with pigment acclimation, which was found to be relevant, were investigated in depth.
\end{abstract}

Keywords: photobioreactor, modeling, microalgae, radiative transfer, spectral effect, red light, incident angle, Chlorella vulgaris

\section{Introduction}

Microalgae are of interest for various applications ranging from the production of high value compounds for cosmetic purposes to new feedstock for biofuel production. ${ }^{1}$ In all the applications, how the photosynthetic microorganisms use light is of prime importance as it governs biological production.

Light use by photosynthetic microorganisms is closely related to light attenuation conditions in the bulk of cultivation systems. Because of the high pigment content of microalgae (around 5\% of biomass dry weight) light is strongly absorbed as it penetrates the culture depth. Three outcomes are possible, usually described by the illuminated fraction $\gamma$, which is the ratio between the depth at which full light attenuation occurs and the reactor depth. In high absorbing conditions, a dark volume appears in the culture system $(\gamma<1)$ with then negative impact on the biomass productivity due to respiration activity of the microalgae. In low absorbing conditions, because not all the light energy is absorbed $(\gamma>1)$, this also results in lowered biomass productivity. ${ }^{2}$ Hence, maximal biomass productivity at a given incident light flux is achieved for a very precise condition of light transfer in the culture volume, where full light attenuation occurs but with no dark volume. This is called the "luminostat regime" $(\gamma=1)$.

Light attenuation conditions are affected by biological parameters such as biomass concentration, spectrumdependent radiative properties of cells and physical parameters mainly related to the incident light flux. To exemplify, Pruvost and Cornet ${ }^{2}$ have investigated the influence on radiative transfer and resulting biomass productivity of some sunlight characteristics such as beam-diffuse radiation partitioning or non-normal incidence of beam radiation on the PBR surface.

A further well-known fact is that kinetically, all the visible photons make the same contribution to photosynthetic rates (reasoning in $\mu \mathrm{mol}_{\mathrm{hv}} / \mathrm{s}$ ) as confirmed by the action spectrum obtained for eukaryotic microalgae. But thermodynamically, photons are absorbed by pigments and then used by photosynthesis with different efficiencies depending on their wavelengths. Because photosynthetic photosystems (PSII and I) 

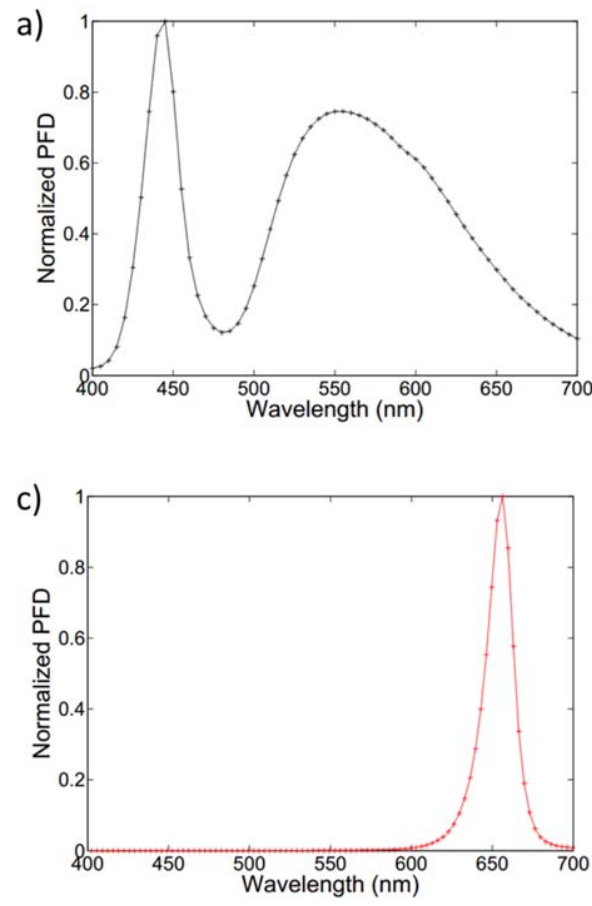

b)

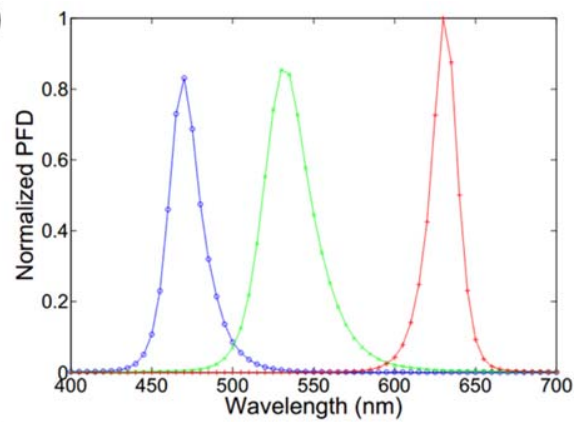

d)

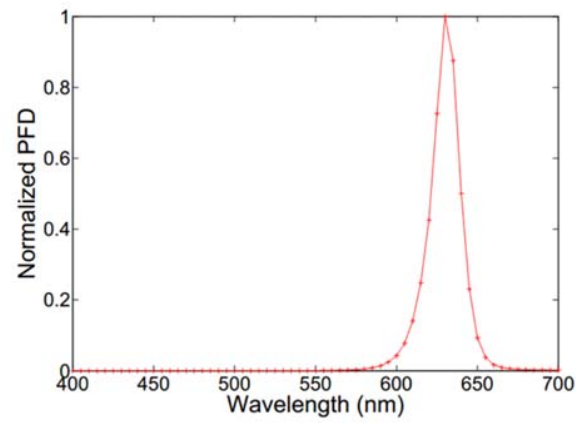

Figure 1. Light emission spectra from LED panels (a: white light for the torus PBR; b: white light for the thin-flat panel PBR as obtained from the combination of blue, green, and red LED emission; c: red light spectrum for the torus PBR; d: red light spectrum for the thin-flat panel PBR).

operate at precise energy levels equivalent to specific photon wavelengths (specifically, red wavelengths of 680 and $700 \mathrm{~nm}$ ), red is used more efficiently than blue light (reasoning in $\mathrm{J} / \mathrm{s}$ or $\mathrm{W}$ ).

There is an abundant literature on the influence of specific radiation spectra in optimizing microalgal growth, but there is generally some confusion between kinetic and thermodynamic effects, or low accuracy in the radiative analysis of the results presented. In all cases, special attention must be paid to the class of oxygenic microorganism considered, the photosynthetic action spectrum of cyanobacteria and eukaryotic microalgae being extremely different in relation to photosystem molecular architectures. For Cylotella nana and Dunaliella terliolecta, it was shown that green light was less efficient than white light, and that blue light was by contrast beneficial. ${ }^{3}$ Figueroa et al. ${ }^{4}$ observed an increase of the growth rate of Porphyra umbilicalis under red compared with blue light. For the cyanobacterium Arthrospira platensis, Wang et al. ${ }^{5}$ showed a higher growth rate under red than under white, green, yellow, and lastly blue light. Recently, Zhao et al. ${ }^{6}$ also investigated the positive effect of red light on Chlorella sp. growth. Farges et al. ${ }^{7}$ investigated the possibility of using red light with Arthrospira platensis for optimal absorption by intracellular pigments and higher thermodynamic efficiency. It was found that modulation of the incident light spectrum affects the pigment content (phycocyanin in this case), a common response to a shift in light spectra. For example, Hess and Tolbert $^{8}$ have shown for Chlorella and Chlamydomonas a decrease in the ratio of chlorophyll a/b and an increase in total chlorophyll content under blue radiation. All these studies emphasize a strong link between light source characteristics and resulting growth. For optimized control of the cultivation system, this link obviously has to be well characterized.
Our study investigates the influence of specific illumination conditions on biomass production in PBRs. White and red incident light spectra were considered, together with non-normal angles of incident radiation as an important feature of solar cultivation in fixed PBRs where non-normal incidence prevails. 9 Experiments in well-controlled conditions were combined with theoretical modeling. The predictive PBR model from Takache et al. ${ }^{10}$ which was developed for Chlamydomonas reinhardtii and for the particular case of normal incidence and white light, was extended in this study to the microalga Chlorella vulgaris CCAP 211-19 grown with different light spectra and incidence angles.

Experiments were conducted in rectangular PBRs. A torus-shaped PBR (named torus PBR) was firstly used with normal incidence and both white and red light to investigate the effect of light spectrum on biomass productivity, and to validate the ability of the proposed model to simulate change in the light emission spectrum. In a second step, the particular case of thin photobioreactors (thickness of $0.007 \mathrm{~m}$ ) was investigated. Because of their high surface-to-volume ratio, these systems enable high volumetric biomass productivity (i.e., high cell density culture). White and red light, and also non-normal incident angle, were applied. Using the model built in the first part of the study, the effects of specific lighting conditions on radiative transfer, pigment adaptation, and biomass productivity could be analyzed.

\section{Materials and Methods}

\section{Selected strain}

The microalga cultivated was C. vulgaris CCAP 211-19. PBR cultures were run in an autotrophic medium containing (g/L): $\mathrm{NH}_{4} \mathrm{Cl} 1.45, \mathrm{MgSO}_{4} \cdot 7 \mathrm{H}_{2} \mathrm{O} 0.281, \mathrm{CaCl}_{2} \cdot 2 \mathrm{H}_{2} \mathrm{O}$ $0.05, \mathrm{KH}_{2} \mathrm{PO}_{4} 0.609, \mathrm{NaHCO}_{3} 1.68$, and $1 \mathrm{~mL}$ of Hutner's 

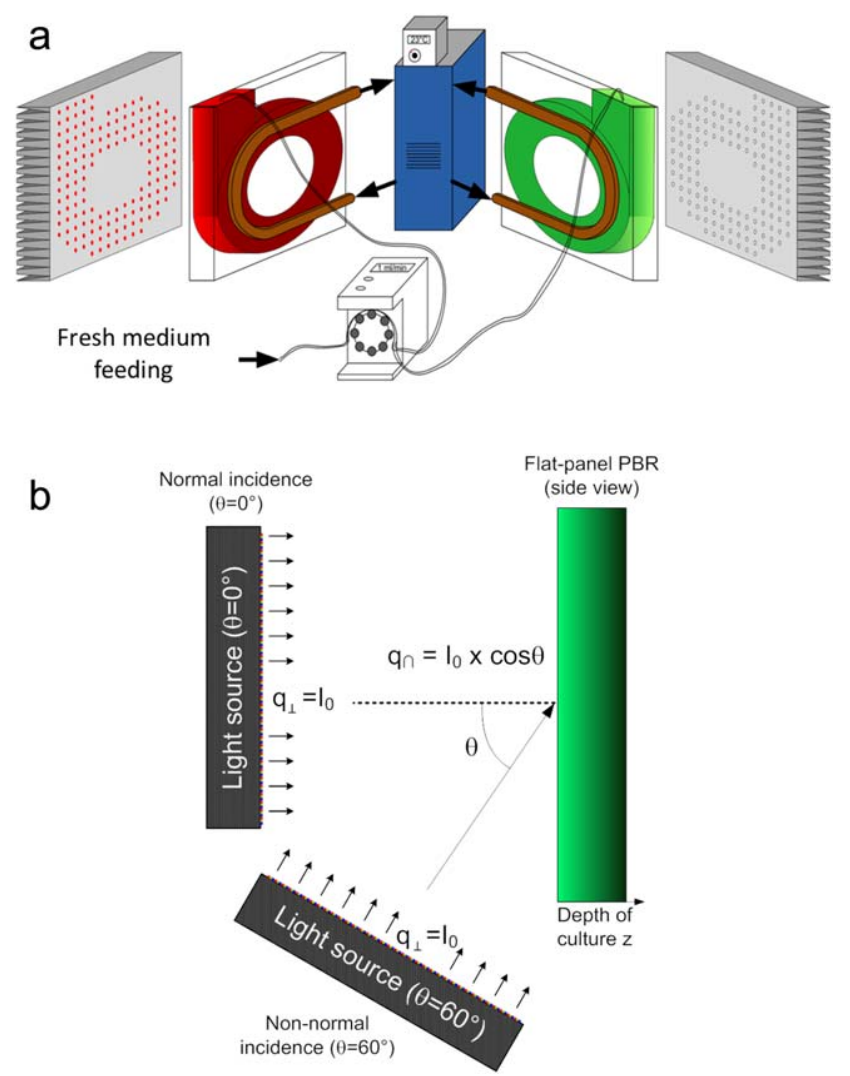

Figure 2. Experimental set-ups for investigating light spectrum and incident angle effects on biomass productivities in PBR (a: torus PBR set-up; b: sketch of thin flat panel PBR for investigation of oblique incidence effect).

trace element solution. Nutrient concentrations were adjusted to avoid mineral limitation and so make sure growth was only light-limited. ${ }^{11}$

\section{Cultivation systems and lighting}

Two PBR types were used for this study: the torus PBR and the thin flat-panel airlift PBR. For each PBR, specific LED panels were designed to produce homogeneous light with high collimation $\left(<10^{\circ}\right)$, so as to guarantee an easy and accurate determination of light transfer in the culture bulk. ${ }^{12}$ Different LED panels were built to apply white or red light (maximum emissive peak at $660 \mathrm{~nm}$ ) on each PBR surface. Light spectra recorded with a hyperspectral radiometer (RAMSES, TriOS optical sensor, Germany) are given in Figure 1.

The torus PBR (Figure 2a) was developed to enable high control over the culture conditions affecting microalgal growth, namely hydrodynamics, lighting and light transfer in culture bulk, and environment $(\mathrm{pH}$ and temperature regulation). This system was found suitable for detailed investigations and for building the photosynthetic growth model. It had a square cross section and could be considered as a "flat panel" with a depth of culture $L=0.04 \mathrm{~m}$. A full description can be found elsewhere. ${ }^{10,13}$ The torus PBR was used to build the growth model of $C$. vulgaris and for a first investigation of the white/red light spectrum effect on biomass productivity. To this end, continuous cultures were run in two identical torus PBRs and in similar conditions, except for the light source. Two LED panels with white and red light spectra respectively were used. The two PBRs were fed in parallel with the same feeding pump and medium so as to guarantee similar feeding flowrates and medium compositions.

A thin flat-panel PBR was used in the second part of the study (Figure 2b). Mixing was obtained by air injection into eight holes regularly spaced all along the base of the PBR. The LED panel was composed of LEDs emitting blue, green, and red light. Each color was controlled individually, so that white light could be obtained by combining the three colors. The influence of the incident angle was investigated by modifying the position of the LED panel with respect to the PBR illuminated surface in order to obtain normal and oblique incidence of LED panel emission (Figure 2b). In all the experiments, the PBR was operated in continuous mode at a constant dilution rate of $0.015 \mathrm{~h}^{-1}$ (i.e., a residence time of $66 \mathrm{~h}$ ).

For both the torus and the thin flat-panel PBR, the temperature and the $\mathrm{pH}$ were regulated at $25^{\circ} \mathrm{C} \pm 1{ }^{\circ} \mathrm{C}$ and $7.5 \pm 0.15$, respectively. The $\mathrm{pH}$ was regulated by an automatic injection of $\mathrm{CO}_{2}$. The temperature was regulated by an external circulation of liquid cooling the torus or the thin flat-panel PBR. The torus and thin flat-panel PBRs were exposed to a photon flux density (PFD) of $200 \mu \mathrm{mol}_{\mathrm{hv}} / \mathrm{m}^{2} / \mathrm{s}$ and $100 \mu \mathrm{mol}_{\mathrm{hv}} / \mathrm{m}^{2} / \mathrm{s}$, respectively. Different values were retained to test the ability of our model to predict PFD influence. The emitting light sources were adjusted to obtain a PFD independent of the light spectrum and incident angle. This was done by measuring the PFD received on the internal PBR surface using a quantum plane sensor (LI-COR LI190-SA and type LI-COR 1400 DataLogger, Lincoln, NE). The sensor was placed on the inner wall of the optical surface of the reactor, and the quasi-homogeneous incident flux was confirmed by measuring PFD at 20 different positions. The power of the LED panels was adjusted in each case so as to obtain the required PFD value.

\section{Analysis}

Dry-Weight Biomass Concentration Measurement and Experimental Productivity Determination. To measure the dry-weight biomass concentration $C_{x}$, a known volume of suspension was filtered on a dry glass fiber filter (dried for $24 \mathrm{~h}$ at $100^{\circ} \mathrm{C}$ ) of pore diameter $0.22 \mu \mathrm{m}$ (GF/F Whatman) and known mass. After filtration, the filter was placed once more in an oven at $100^{\circ} \mathrm{C}$ for $24 \mathrm{~h}$ and reweighed. The ratio of the mass difference to the filtered volume gave the dry weight of the analyzed suspension.

Dry-weight biomass concentration was used to determine biomass productivity. For a perfectly mixed PBR operated in continuous mode and at steady-state, the growth rate is equal to the biomass volumetric productivity $P_{x}:{ }^{2}$

$$
\left\langle r_{x}\right\rangle=P_{x}=C_{x} / \tau_{p}=C_{x} \cdot D,
$$

with $\left\langle r_{x}\right\rangle$ the mean volumetric biomass production rate in the system, and $\tau_{p}$ the residence time resulting from the liquid flow rate of the feed (fresh medium) (with $\tau_{p}=1 / D$, where $D$ is the dilution rate). Each experimental point is an average of three measurements obtained at steady state. ${ }^{10}$

Pigment Content Estimation. Pigment content was estimated by spectrophotometric analysis. A known volume of suspension was centrifuged and the supernatant removed. A known volume of methanol was added and the centrifuged 
cells were re-suspended in this solvent. After $1 \mathrm{~h}$ in a dark oven at $44^{\circ} \mathrm{C}$, the sample was centrifuged again and the supernatant was analyzed by spectrophotometry (Jasco V630, Jasco, France). Three replicates were prepared, and chlorophyll-a, b, and photoprotective carotenoids (PPC) were assayed using the following relationships: ${ }^{14,15}$

$$
C_{\mathrm{Chl}_{\mathrm{a}}}=\left[-8.0962 \times\left(A_{652}-A_{750}\right)+16.5169 \times\left(A_{665}-A_{750}\right)\right] \times V_{2} / V_{1} \times L_{p},
$$

$C_{\mathrm{Chl}_{\mathrm{b}}}=\left[27.4405 \times\left(A_{652}-A_{750}\right)-12.1688 \times\left(A_{665}-A_{750}\right)\right] \times V_{2} / V_{1} \times L_{p}$,

$$
C_{\mathrm{PPC}}=4.0000 \times\left(A_{480}-A_{750}\right) \times V_{2} / V_{1} \times L_{p},
$$

where $V_{2} / V_{1}$ is the ratio of the solvent volume to the suspension volume, and $L_{p}$ the cell path length $(1 \mathrm{~cm})$.

\section{Theoretical Considerations}

\section{Overview of light-limited growth modeling in a PBR}

In all the experiments, cultivation conditions were set so as to obtain light-limited growth conditions, ${ }^{2,10}$ where biomass production rate is a function only of the light received (no mineral limitations, optimal $\mathrm{pH}$, and temperature values). This enabled us to apply the approach described in Takache et al. ${ }^{10}$ to model light-limited growth in photobioreactors for the microalga Chlamydomonas reinhardtii. This model is based on coupling a kinetic photosynthetic growth model and a radiative transfer model to describe the light attenuation within the PBR bulk as a function of parameters affecting the light transfer: biomass concentration, microalgal radiative properties, and light emission characteristics (spectrum, PFD, and incident angle). The coupling between radiative and kinetic growth models allows us to calculate the resulting mean volumetric biomass production rate $\left\langle r_{x}\right\rangle$ and ultimately the biomass concentration and productivity.

\section{Kinetic growth model for Chlorella vulgaris}

In light-limited conditions, the kinetic growth model relates the heterogeneous light radiation field in the PBR to the local photosynthetic growth rate. This thermokinetic coupling formulation has long been adopted by the authors for the crucial role played by the local specific rate of radiant energy absorbed $\mathcal{A}$ in photoreactor or photobioreactor modeling. ${ }^{2,10,16-19}$ Until now, and especially in Takache et al., ${ }^{10}$ the general form of this coupling has taken the form $J_{i}=\overline{\phi_{i}^{\prime}} \rho$ $\mathcal{A}$ (for any component $i$ ) in which the energy yield $\rho$ was assumed to decrease following a law involving the local irradiance $G$, written $\rho=\rho_{M} \frac{K}{K+G}$ (leading to a classical hyperbolic law of photosynthesis with $G$ ). The authors recently found it preferable to represent the decrease in photosynthetic rates also from the local specific rate of photon absorption $\mathcal{A}$ instead of $G$. The specific rate of photon absorption $\mathcal{A}=E_{a} . G$ represents the light effectively absorbed by the cells, which is a combination of the light received (irradiance $G$, in $\mu \mathrm{mol}_{\mathrm{h} v} / \mathrm{m}^{2} / \mathrm{s}$ ), and the ability of the cells to absorb light (absorption coefficient $E_{a}$ in $\mathrm{m}^{2} / \mathrm{kg}$ ). Because light absorption by cells depends on their pigment content, which is highly variable, the rate of photon absorption was found more representative (both for kinetic modeling and cell regulation mechanisms) than considering the irradiance value alone. The formulation proposed by Takache et al. ${ }^{10}$ was then modified by considering the specific rate of light energy absorption $\mathcal{A}$ in place of the irradiance $G$ in the formulation of the energy yield $\rho$, so that $\rho=\rho_{M} \frac{K}{K+\mathcal{A}}$.

Because of the use of different light emission spectra, a spectral calculation was also performed. The specific rate of photon absorption noted $\mathcal{A}$ (in $\mu \mathrm{mol}_{\mathrm{h}} / \mathrm{s} / \mathrm{kg}$ ) was obtained by integrating over the PAR region the product of the spectral values of local irradiance $G_{\lambda}$ and of microalgal mass absorption coefficient $E_{a \lambda}$ :

$$
\mathcal{A}=\int_{\mathrm{PAR}} E_{a \lambda} G_{\lambda} \mathrm{d} \lambda .
$$

These two-point modifications (spectral averaging calculation of $\mathcal{A}$ and its use in the calculation of $\rho$ ) in the formulation of the coupling between local light absorption and kinetic rates were proposed for the first time in the $\mathrm{PhD}$ thesis of Dauchet ${ }^{19}$ for $C$. reinhardtii, and are published here for the first time in a article using Chlorella as photosynthetic microorganism. We note that this formulation is particularly well suited to situations where the pigment content of the cells is strongly affected by culture conditions, as is the case for example when mineral starvation is used to increase the lipid production rate in PBR for producing biofuel precursors. ${ }^{20}$

Except for this change in the definition of $\rho$, considered to vary with the true light energy absorbed $\mathcal{A}$ instead of the hypothetical available light energy $G$, the formulation proposed by Takache et al. ${ }^{10}$ was kept unchanged. This formulation was found useful for representing relevant features of the photosynthetic response of microalgae to light such as the progressive saturation of growth with light, and the decrease in respiration activity with respect to light as an adaptive process of the cell energetic. $^{20-22}$ The formulation expresses the photosynthetic growth from the local specific rate of oxygen production or consumption $J_{\mathrm{O}_{2}}$ considered here at the scale of intracellular organelles, close to the primary photosynthetic and respiration events:

$J_{\mathrm{O}_{2}}=\left[\rho \overline{\phi_{\mathrm{O}_{2}}^{\prime}} \mathcal{A}-\frac{J_{\mathrm{NADH}_{2}}}{v_{\mathrm{NADH}_{2}-\mathrm{O}_{2}}} \cdot \frac{K_{\mathrm{r}}}{K_{\mathrm{r}}+\mathcal{A}}\right]=\left[\rho_{\mathrm{M}} \frac{K}{K+\mathcal{A}} \overline{\phi_{\mathrm{O}_{2}}^{\prime}} \mathcal{A}-\frac{J_{\mathrm{NADH}_{2}}}{v_{\mathrm{NADH}_{2}-\mathrm{O}_{2}}} \cdot \frac{K_{\mathrm{r}}}{K_{\mathrm{r}}+\mathcal{A}}\right]$,

where $\rho_{M}$ is maximum energy yield for photon conversion, $\overline{\phi_{O_{2}}^{\prime}}$ is the mole $\mathrm{O}_{2}$ quantum yield for the $Z$ scheme of photosynthesis, $K$ the half-saturation constant for photosynthesis, $J_{N_{A D H}}$ the specific rate of cofactor regeneration on the respiratory chain, linked to oxygen consumption by the stoichiometric coefficient $v_{\mathrm{NADH}_{2}-O_{2}}$ (the stoichiometric coefficient of cofactor regeneration on the respiratory chain), and $K_{r}$ is a saturation constant describing the inhibition of respiration in light.

As a direct result of the light distribution within the culture, the kinetic relation is of the local type. This implies calculating the corresponding mean value by averaging over the total culture volume $V_{R}$ :

$$
\left\langle J_{O_{2}}\right\rangle=\frac{1}{V_{R}} \iiint_{V_{R}} J_{O_{2}} d V .
$$

For cultivations systems with Cartesian one-dimensional light attenuation as in the case in our study (i.e., flat panel geometries), this reduces to a simple integration along the depth of culture $z$ : 


$$
\left\langle J_{O_{2}}\right\rangle=\frac{1}{L} \int_{z=0}^{z=1} J_{O_{2}} d z
$$

where $L$ is the depth of the reactor.

Finally, once $\left\langle J_{O_{2}}\right\rangle$ is known, the mean volumetric biomass growth rate $\left\langle r_{x}\right\rangle$ can be derived directly using the stoichiometry relating oxygen and biomass productions:

$$
\left\langle r_{x}\right\rangle=\frac{\left\langle J_{O_{2}}\right\rangle C_{x} M_{x}}{v_{O_{2}-X}}
$$

where $M_{X}$ is the C-molar mass for the biomass and $v_{O_{2}-X}$ is the stoichiometric coefficient of the oxygen production.

Finally, once the mean volumetric growth rate is known, the resolution of the mass balance equation for biomass lets us calculate the biomass concentration and productivity as a function of control parameters (lighting conditions and dilution rate $D$-or residence time $\tau_{p}=1 / D$-resulting from the liquid flow rate of the feed):

$$
\frac{d C x}{d t}=\left\langle r_{x}\right\rangle-\frac{C_{x}}{\tau_{p}}
$$

\section{Modeling of radiative transfer and determination of radiative properties}

Solving Eq. (6) implies determining the field of the specific rate of photons absorption $\mathcal{A}$, which is obtained from the radiative transfer modeling. All the PBRs used in this study present light attenuation along only one main direction (i.e., the depth of culture $z$ ) so we can apply the two-flux model already shown to be efficient in several studies. ${ }^{10,23-25}$ A complete description can be found in Pottier et al. ${ }^{12}$ and in Pruvost et al. ${ }^{26}$ The two-flux model was expressed in this study as a function of the wavelength and the incident angle $\theta$, to take into account the general case of oblique irradiation with any incident light spectrum. For all the PBRs and conditions tested, the irradiance field was then given by the general formula (collimated incident light):

$$
\frac{G_{\lambda}}{q_{\lambda}}=\frac{2}{\cos (\theta)} \frac{\left[R_{S}\left(1+\alpha_{\lambda}\right) \exp \left(-\delta_{\lambda} L\right)-\left(1-\alpha_{\lambda}\right) \exp \left(-\delta_{\lambda} L\right)\right] \exp \left[\delta_{\lambda} z\right]+\left[\left(1+\alpha_{\lambda}\right) \exp \left(\delta_{\lambda} L\right)-R_{S}\left(1-\alpha_{\lambda}\right) \exp \left(\delta_{\lambda} L\right)\right] \exp \left[-\delta_{\lambda} z\right]}{\left(1+\alpha_{\lambda}\right)^{2} \exp \left(\delta_{\lambda} L\right)-\left(1-\alpha_{\lambda}\right)^{2} \exp \left(-\delta_{\lambda} L\right)+R_{S}\left(1-\alpha_{\lambda}\right)^{2}\left[\exp \left(-\delta_{\lambda} L\right)-\exp \left(\delta_{\lambda} L\right)\right]} .
$$

Here, $\mathrm{q}_{\lambda}$ is the PFD measured perpendicularly to the illuminated surface, $\alpha_{\lambda}=\sqrt{\frac{E_{a \lambda}}{\left(E_{a \lambda}+2 b_{\lambda} E_{s \lambda}\right)}}$ is the spectral linear scattering modulus, and $\delta_{\lambda}=\frac{\alpha_{\lambda} C_{x}}{\cos (\theta)}\left(E_{a \lambda}+2 b_{\lambda} E_{s \lambda}\right)$ is the spectral two-flux extinction coefficients. $E_{a \lambda}$ and $E_{s \lambda}$ are spectral values of mass absorption and mass scattering coefficients for the cultivated photosynthetic microorganism respectively, and $b_{\lambda}$ is the back-scattered fraction.

Equation 11 is given for a non-transparent, reflecting rear side, as obtained for the torus PBR, which presents a stainless steel plate for cooling at the rear of the reactor $\left(R_{S}=0.52\right)$. For the thin flat-panel airlift $\mathrm{PBR}$, the rear side is transparent $\left(R_{S}=0\right)$ and the Eq. (11) simplifies to:

$$
\frac{G_{\lambda}}{q_{\lambda}}=\frac{2}{\cos (\theta)} \frac{\left(1+\alpha_{\lambda}\right) \exp \left[-\delta_{\lambda}(z-L)\right]-\left(1-\alpha_{\lambda}\right) \exp \left[\delta_{\lambda}(z-L)\right]}{\left(1+\alpha_{\lambda}\right)^{2} \exp \left[\delta_{\lambda} L\right]-\left(1-\alpha_{\lambda}\right)^{2} \exp \left[-\delta_{\lambda} L\right]} .
$$

Equations 11 (torus PBR) and 12 (thin-flat panel airlift PBR) give a spectral light attenuation profile (i.e., function of light wavelength). As already described elsewhere, ${ }^{12}$ they can be simplified for a spectrally averaged resolution to reduce calculation time. This simplification will be tested later in this work. Except when cited, the spectral formulation was applied in calculation.

The accurate prediction of the light attenuation conditions in the culture volume implies determining spectral radiative properties of microalgal suspension $\left(E_{a \lambda}, E_{s \lambda}, b_{\lambda}\right)$. These properties were determined using the experimental method described in Kandilian et al. ${ }^{27}$ Because of the wide variation in pigment content, a complete database of spectral radiative properties was also reconstructed theoretically as a function of the pigment content covered in all the experiments. Because of the spherical shape of $C$. vulgaris, these radiative properties were in this case calculated from Lorenz-Mie theory, ${ }^{12,28}$ The complete method is fully described by Dauchet et al. ${ }^{29}$ and was previously applied to calculate radiative properties of Chlamydomonas reinhardtii. ${ }^{10}$ The input parameters are the pigment contents and the size distributions of the cells. These parameters were determined experimentally. The size distribution of $C$. vulgaris CCAP 211/19 was measured in Soulies et al. ${ }^{30}$ It was shown that $C$. vulgaris cells were near-spherical (Feret ratio $\approx 0.82$ ) with an average radius of $2 \mu \mathrm{m}$. Size distribution of a log-normal type was found, with a standard deviation of 1.218 .

The field of photon absorption rates [i.e., $\mathcal{A}(\mathrm{z})$ ] was used to determine the illuminated fraction $\gamma$, whose relevance in PBR engineering is described elsewhere. ${ }^{2,31,32}$ Schematically, the culture volume $V_{r}$ is split into two zones: an illuminated zone of volume $V_{\text {light }}$ and a dark zone of volume $V_{\text {dark }}$. The illuminated fraction $\gamma$ is then given by the depth of culture $z_{c}$ where the value of specific rate of photons absorption for compensation $\left(z_{c}\right)=\mathcal{A}_{c}$ is obtained, $\mathcal{A}_{c}$ being the minimum value required to obtain a positive photosynthetic growth $\left(J_{O_{2}}=0\right.$ for $\mathcal{A}=\mathcal{A}_{c}$ in Eq. (6), see later for its determination). In the case of cultivation systems with onedimensional light attenuation, we obtain:

$$
\gamma=\frac{V_{\text {light }}}{V_{R}}=\frac{z_{c}}{L} .
$$

A $\gamma$ value below 1 indicates that all the light available for photosynthesis is absorbed by the culture. Conversely, when the illuminated fraction is greater than 1 (a hypothetical representation because at maximum $V_{\text {light }}=V_{r}$ ), some of the light is transmitted.

\section{Results and Discussion}

\section{Continuous cultivation in a torus PBR under red and white light}

Biomass Productivity as a Function of Dilution Rate. A set of experiments was conducted in the torus PBR for a first characterization of the effect of light spectrum on biomass 

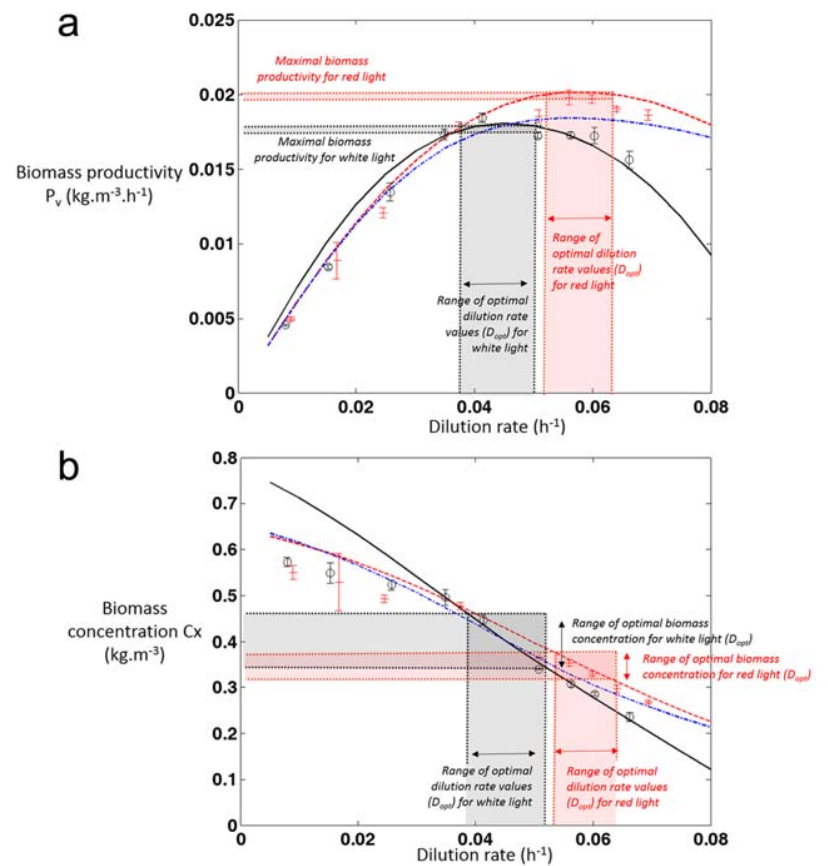

C

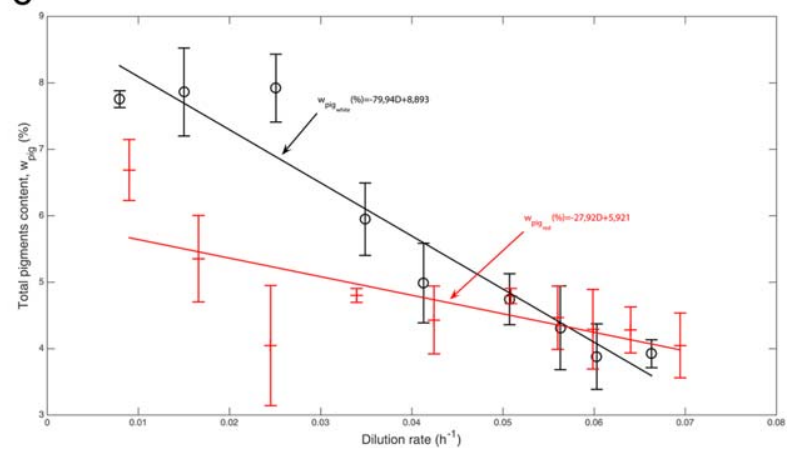

Figure 3. Volumetric biomass productivity (a), biomass concentration (b), and pigment content (c) as a function of the dilution rate for $C$. vulgaris culture in torus PBR (circle and plus marks are experimental data for white and red light, respectively; solid and dashed lines are theoretical predictions; the dashdot line is for spectrally averaged resolution-see text for details).

growth, and to obtain experimental values to build the kinetic growth model for $C$. vulgaris. It consisted of continuous cultures for various dilution rates in chemostat mode, and for white and red light spectra giving the same incident PFD of $200 \mu \mathrm{mol} / \mathrm{m}^{2} / \mathrm{s}$.

Figure 3 shows $C$. vulgaris biomass concentration and corresponding volumetric productivity as a function of dilution rate. The progressive decrease in biomass concentration with the increase in dilution rate reduced the effect of light attenuation in the PBR bulk, resulting in an increase in biomass productivity up to a peak value. ${ }^{2,32}$ Maximal productivity was then obtained for an optimal value of the dilution rate, also corresponding to an optimal biomass concentration leading to optimal light attenuation conditions in the culture volume (i.e., luminostat regime corresponding to full light absorption with no dark zone in the culture bulk). The decrease of biomass productivity for higher dilution rate is related to lower light attenuation conditions, which induces a higher light amount received per cell, that is, a high rate of photon absorption $\mathcal{A}$. We know that photosynthetic conver- sion (and more generally metabolism of photosynthetic microorganisms) is strongly affected by an excess of light. This can induce specific biological responses such as pigment loss or even photoinhibition, which can induce culture drift or loss of efficiency, resulting in a loss of biomass productivity and potentially impairing culture stability. ${ }^{33-35}$ We can note that in practice, such conditions of low light attenuation prove very difficult to maintain, and there is then a high risk of culture washout when operating in continuous mode.

Influence of Light Spectrum on Biomass Productivity. Optimization of the incident light spectrum is often envisaged for better microalgal growth in a PBR..$^{5,36-39}$ We were here interested in quantifying the increase that could be obtained for $C$. vulgaris growth in a PBR, by comparing the usually applied white light emission spectrum with red. In our experiments (Figure 3), biomass productivity was increased with the red light spectrum, but only to a modest extent (around 10\%). Modification of the light spectrum appeared mainly to influence the conditions leading to maximal biomass productivities. Optimal values of the dilution rate were found in the range $0.038-0.048 \mathrm{~h}^{-1}$ (residence times of $21-27 \mathrm{~h}$ ) and $0.052-0.063 \mathrm{~h}^{-1}$ (residence times of 16-20 h) for white and red light respectively. This corresponded to widely different optimal biomass concentrations, in the range $0.35-0.48 \mathrm{~kg} / \mathrm{m}^{3}$ and $0.32-0.38 \mathrm{~kg} / \mathrm{m}^{3}$ for white and red light, respectively. Hence, a higher value of optimal dilution rate was obtained in red light, corresponding to lower optimal biomass concentration.

It was also interesting to compare the relation between biomass concentration and dilution rate as a function of light spectrum. Closely similar patterns were obtained for dilution rate values lower than optimal. As this also corresponds to dark volumes in the culture bulk, we can conclude that the incident light spectrum had only a slight effect in the conditions of full light attenuation, for both biomass concentration and for resulting biomass productivity. The light spectrum effect became significant when optimal dilution rate values were reached and overtaken, that is, when the dark volume vanished in the PBR. A higher biomass concentration was then obtained for a given dilution rate under red light. As observed previously, this also corresponded to a slightly higher biomass productivity. For a dilution rate higher than optimal, a lower decrease in biomass concentration was obtained with the red light spectrum. As a result, this allowed us to apply higher dilution rates than with white light before culture became unstable and washed out.

Influence of Light Spectrum on Pigment Content. Figure $3 \mathrm{c}$ presents pigment content as a function of operating conditions. For both white and red lights, the pigment content decreased with increasing dilution rates (i.e., decrease in biomass concentration and light attenuation), indicating pigment adaptation to conditions of light attenuation in the PBR. The pigment content was found to be higher with white light, with a marked difference for high light attenuation (low dilution rates). For low light attenuation conditions $\left(D>D_{o p t}\right)$, pigment contents were found to be closely similar for the two light spectra.

All these results emphasize a strong relation between pigment content and light received, especially in the conditions of high light attenuation. It is well known that photosynthetic microorganisms demonstrate photoacclimation. ${ }^{40,41}$ Hence an increase in pigment content to partly offset increased light attenuation in the culture volume is no surprise. In our case, 
however, a marked loss of pigment content was observed, around 1.5- to 2-fold variations over our range of dilution rates. We also note that the distribution between various pigments (Chla, Chlb, and photoprotective carotenoids) stayed almost constant whatever the conditions investigated (light spectrum, dilution rate): $66 \% \pm 3 \%$ chlorophyll a, $17 \% \pm 3 \%$ chlorophyll b, and $17 \% \pm 1 \%$ photoprotective carotenoids (values relative to total pigment content). Cultures did not, therefore, exhibit any significant acclimation to the light spectrum, as might at first sight have been expected, and as already observed elsewhere. ${ }^{42}$ A different light spectrum resulted in different pigment content, but this seemed in our case related more to light attenuation conditions that differed in red and white light. We also note that this near-constant distribution between chlorophylls and carotenoids confirms that the culture did not suffer from nutrient limitation, which is known to result in significant changes in the chlorophyllto-carotenoids ratio. ${ }^{43}$

Given the marked variation in pigment content with operating conditions, radiative properties of $C$. vulgaris were determined. Corresponding values are given in Supporting Information. These values were used in all the subsequent calculations. We note that these values could be easily applied in other studies, assuming the values of the parameters affecting radiative properties such as cell size distribution and distribution among pigments are similar to those obtained here.

Kinetic Growth Model for Chlorella vulgaris. Following Takache et al., ${ }^{10}$ the parameters in Eq. (6) may be divided into two groups. The first can be determined using a fully predictive method $\left(\rho_{M}, \overline{\phi_{O_{2}}^{\prime}}, E_{a}\right.$, and $\left.v_{\mathrm{NADH}_{2}-O_{2}}\right)$, whereas the second need to be estimated from experimental data $\left(J_{\mathrm{NADH}_{2}}, K\right.$, and $\left.K_{r}\right)$.

As a radiative property, the spectral mass absorption coefficient was determined in the previous section. The value of the mean mole quantum yield $\overline{\phi_{O_{2}}^{\prime}}$ and $v_{\mathrm{NADH}_{2}-\mathrm{O}_{2} \text { was }}$ obtained by establishing a structured stoichiometric equation of the photosynthetic growth, obtained from the elemental composition of the biomass produced. This ultimately enabled us to write the stoichiometric equation of biomass synthesis $^{44}$ :

$$
\begin{aligned}
& \mathrm{HCO}_{3}^{-}+0.4940 \mathrm{H}_{2} \mathrm{O}+0.1590 \mathrm{NH}_{4}^{+}+0.006 \mathrm{SO}_{4}^{2-} \\
& +0.007 \mathrm{PO}_{4}^{3-} \longrightarrow \mathrm{CH}_{1.750} \mathrm{O}_{0.413} \mathrm{~N}_{0.159} \mathrm{~S}_{0.006} \mathrm{P}_{0.007} \\
& +1.1295 \mathrm{O}_{2}+0.8740 \mathrm{HO}^{-}
\end{aligned}
$$

This global stoichiometric equation gives us a structured equation, ${ }^{44}$ involving ATP and reduced cofactors, as presented by Cornet and Dussap, ${ }^{45}$ enabling us to calculate the theoretical value of the oxygen molar quantum yield as a unique value for any microorganism $\overline{\phi_{O_{2}}^{\prime}}$. This leads to:

$$
\overline{\phi_{\mathrm{O}_{2}}^{\prime}}=1.1 \times 10^{-7} \mathrm{~mol}_{\mathrm{O}_{2}} / \mu \mathrm{mol}_{h v} \text {. }
$$

Trivially, considering the stoichiometry between $\mathrm{NADH}_{2}$ and $\mathrm{O}_{2}, v_{\mathrm{NADH}_{2}-\mathrm{O}_{2}}=2$. Likewise, the general value for the maximum energy yield of light energy conversion $\rho_{M}=0.8$ was used according Cornet and Dussap. ${ }^{45}$

Missing parameters $J_{\mathrm{NADH} 2}, K$, and $K_{r}$, were determined from experiments: the results obtained in the torus PBR with white incident light spectrum were used. From Eq. (6), we note that the parameter $K_{\mathrm{r}}$ is not independent and can be linked to the specific rate of cofactor regeneration on the respiratory chain by the definition of the compensation point of photosynthesis. This compensation point is obtained for a value of rate of photon absorption leading to null net oxygen production (i.e., $J_{\mathrm{O}_{2}}=0$ ). We can rewrite Eq. (6) as:

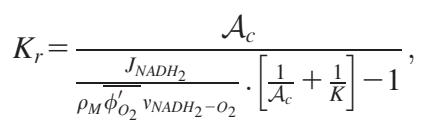

with $\mathcal{A}_{c}$ the value of specific rate of photon absorption at the compensation point.

The value of $\mathcal{A}_{c}$ can also be estimated from the experiments. Maximal biomass productivity was obtained for luminostat regime $(\gamma=1)$ meaning that full light attenuation conditions with no dark zone are achieved in the culture bulk. Following Takache et al., ${ }^{10,32}$ this implies that the minimum local value of specific rate of photon absorption in the culture volume cannot be lower than $\mathcal{A}_{c}$. For a flat PBR illuminated from one side, this minimum value is obtained at $z=L$. Thus, $\mathcal{A}_{c}$ can be estimated by determining the attenuation profile of specific rate of photon absorption (Eqs. 5 and 11) for the biomass concentration that leads to maximal biomass productivity.

This method was applied to our results. Because of the experimental uncertainty, we took a range of operating conditions roughly corresponding to the experimental maximal biomass productivities (Figure 3). We then considered that an optimal value of biomass concentration was achieved in the range $C_{x} \approx 0.35-0.48 \mathrm{~kg} / \mathrm{m}^{3}$ (4\%-4.8\% in pigments) and $C_{x} \approx 0.32-0.38 \mathrm{~kg} / \mathrm{m}^{3}$ (4\%-4.5\% in pigments) for the white and red light spectra, respectively. Corresponding median values were $C_{x}=0.41 \mathrm{~kg} / \mathrm{m}^{3} \quad(4.4 \%$ in pigments $)$ and $C_{x}=0.35 \mathrm{~kg} / \mathrm{m}^{3}$ (4.25\% in pigments) for the white and red light spectra, respectively.

Based on these values of optimal operating conditions, profiles of attenuation of the rate of photon absorption were estimated (Figure 4, spectral radiative properties taken from the Table in Supporting Information). We note that different profiles of rates of photon absorption in the culture bulk were obtained, as a result of differences in biomass and pigment concentrations obtained at maximal productivities for both white and red lights. However, similar values of photon absorption rates were obtained at the bottom of the PBR (Figure 4b). By definition of the light attenuation conditions corresponding to maximal productivities (i.e., full light attenuation with no dark zone), this value as obtained at $z=L$ should be in the range of $\mathcal{A}_{c}$. Based on this observation, Figure 4 presents attenuation profiles of the rate of photon absorption for median and boundary values of the range of conditions identified previously. Values of the rate of photon absorption for $z=L$ were found in the range $2,800-4,500 \mu \mathrm{mol}_{\mathrm{hv}} / \mathrm{kg} / \mathrm{s}$ (3,650 for median values of operating conditions) and 3,500$5,900 \mu \mathrm{mol}_{\mathrm{h} v} / \mathrm{kg} / \mathrm{s}$ (4,800 for median values) for white and red light, respectively.

Based on this estimation of $\mathcal{A}_{c}$, the parameters of the growth model were obtained by minimizing the relative error between experimental data and theoretical predictions (Nelder-Mead method, fminsearch routine in Matlab software). The kinetic growth model being fully dependent on the light attenuation field resolution, two cases were considered. The simplest resolution was obtained using spectrally averaged radiative properties, which reduces calculation time. ${ }^{12}$ Because of the consideration in this study of two different emission light spectra (i.e., white and red), radiative transfer was also spectrally solved. This leads to 

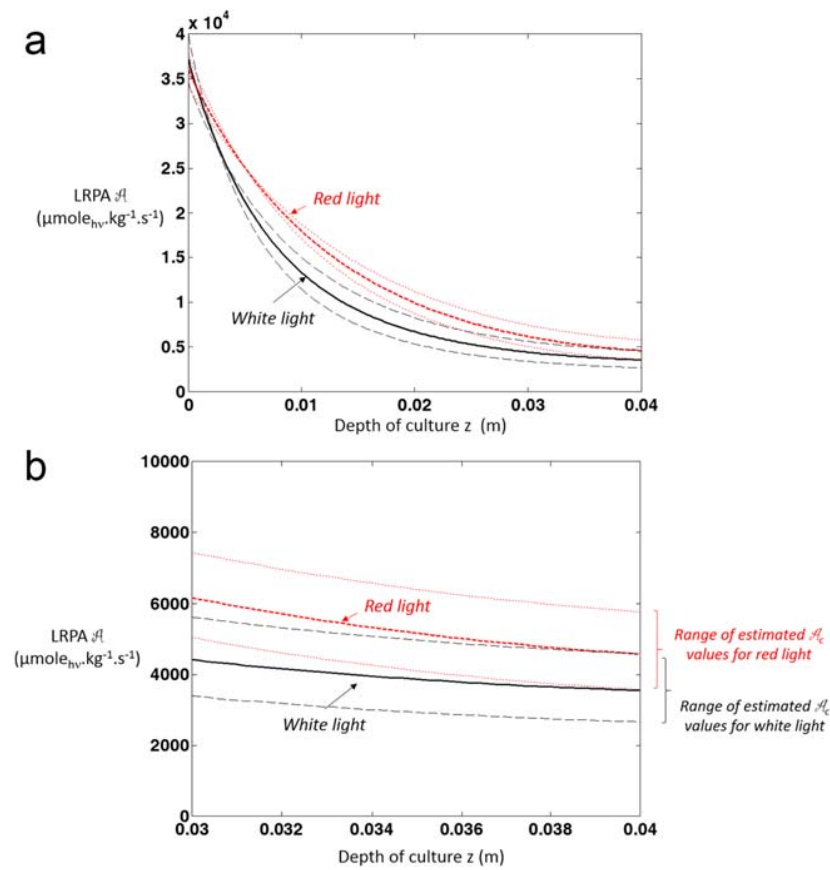

Figure 4. Evolution of the local rate of photon absorption $\mathcal{A}$ along the PBR depth (bold solid and dashed lines are for white and red light spectra, respectively; thin lines were obtained for boundary values of the range of optimal biomass concentration and pigment contents leading to maximal productivities as identified in Figure 3). A focus is given in (b) for the lower values of $\mathcal{A}$ as obtained in the bottom the PBR (total depth $L=0.04 \mathrm{~m}$ ).

different light attenuation profiles and so different theoretical values of predicted growth rates. Hence, the minimization procedure leads to different values of the kinetic growth model parameters. Values are summarized in Table 1 for both cases.

The slight discrepancy in $\mathcal{A}_{c}$ values between those estimated from experiments and those obtained from the kinetic growth model calls for comment. Values from the kinetic growth model $\left(\mathcal{A}_{c}=2,800 \mu \mathrm{mol}_{\mathrm{h} v} / \mathrm{kg} / \mathrm{s}\right.$ and $\mathcal{A}_{c}=1,500$ $\mu \mathrm{mol}_{\mathrm{h} v} / \mathrm{kg} / \mathrm{s}$ for spectral and spectrally averaged resolution, respectively) were found to be slightly below values estimated from the calculation of the rate of photon absorption attenuation profiles. This is consistent with the assumption that the minimum local value of specific rate of energy absorption in the culture volume as achieved for maximal biomass productivity cannot be lower than $\mathcal{A}_{c}$ (no dark volume). We note that for the ideal condition of no light reflection in the bottom of the PBR where minimum $\mathcal{A}$ value is achieved, the condition of $\mathcal{A}(z=L)=\mathcal{A}_{c}$ should be obtained at maximal biomass productivity (Takache et al., 2012). Experiments were, however, conducted in a PBR equipped with a stainless steel rear panel. The light reflection thus induced a slight increase in irradiance in the illuminated volume. Consequently, the minimum value of the rate of photons absorption as obtained at $z=L$ did not correspond exactly to the value $\mathcal{A}_{c}$ at the compensation point, but was slightly higher.

Prediction of Biomass Productivity and Model-Based Analysis. Comparison with experimental data. Modeling was used to predict change of biomass concentration as a function of dilution rate and productivities in torus PBRs for both white and red light spectra. The marked changes in pigment content with operating conditions were considered by assuming to a first approximation a linear evolution with the dilution rate. These empirical relations are given in Figure $3 c$ for both light spectra. They were used to calculate (for each condition) the corresponding radiative properties from the Table in Supporting Information.

The results of model prediction for both light spectra are given in Figures 3a,b. The relative error with experimental data is around $7 \%$, and the model was able to represent effects of light spectrum on biomass productivity. The main discrepancy between the model prediction and the experimental data is found for biomass concentration at low values of dilution rate, for both white and red light spectra. This problem was already observed by Takache ${ }^{32}$ and could be explained by an inaccurate formulation of the respiration activity in the growth model (Eq. 6), causing us to overestimate the biomass concentration when high light attenuation occurs with significant dark zones in which respiration activity prevails. This is clearly a weakness of our kinetic formulation, but considering that the deviation was kept below $10 \%$, the model prediction could be considered satisfactory. The model with its set of parameters as defined in Table 1 was, thus, retained for the rest of the study.

Influence of pigment acclimation and spectrally averaged resolution of light transfer attenuation. The model was used to further investigate the experimental results. Firstly the influence of pigment acclimation on resulting biomass productivity was investigated, assuming a constant pigment content in the calculation, here set at an average value of $4 \%$. An error of $12 \%$ in biomass productivity predictions was obtained instead of $7 \%$ considering pigment content evolution (data are not shown in Figure 3 because evolutions similar to those with pigment acclimation were obtained). This illustrates the influence of pigment acclimation of C. vulgaris on resulting productivity, by modifying light attenuation conditions in the PBR bulk and thereby in the resulting photosynthetic growth. Ideally, pigment acclimation has to be considered in the modeling approach for accurate predictions.

Radiative transfer can be solved spectrally by considering evolutions as a function of wavelengths of radiative properties (see Table in Supporting Information) and emitted light (Figure 1), or by using spectrally averaged values. The influence of this last assumption was tested, and the results were added to predictions obtained with spectral resolution in Figure 3. As expected, because the PFD was equal, exactly the same predictions were obtained for white and red light emission spectra using a spectrally averaged resolution (resulting curves are superimposed in Figure 3). The model proved unable to predict the effect of a change of light emission spectrum (for the same PFD). We note, however, that the error in the prediction remained acceptable (8.5\%). This is explained by the use of a different set of growth kinetic parameters for spectral and spectrally averaged resolution (see the previous section on model parameter determination, Table 1). By considering the same set of growth kinetic parameters as for the spectral resolution, an error of $12 \%$ was obtained. Hence, except when investigating the specific influence of light spectrum as here, a spectrally averaged resolution can be used as an effective way to reduce 
Table 1. Summary of the Growth Model Parameters for Chlorella vulgaris

\begin{tabular}{|c|c|c|c|c|}
\hline Parameter & $\begin{array}{l}\text { Value (obtained from spectral } \\
\text { resolution) }\end{array}$ & $\begin{array}{l}\text { Value (obtained from spec- } \\
\text { trally averaged resolution) }\end{array}$ & Unit & $\begin{array}{l}\text { Method of identification } \\
\text { (see text for details) }\end{array}$ \\
\hline$\rho_{\mathrm{M}}$ & 0.8 & 0.8 & - & Predictive determination \\
\hline$J_{\mathrm{NADH}_{2}}$ & $1.8 \times 10^{-3}$ & $1.8 \times 10^{-3}$ & $\mathrm{~mol}_{\mathrm{NADH}_{2}} / \mathrm{kg}_{x} \mathrm{~s}$ & \\
\hline$\underline{v_{0_{2}}-X}$ & 1.13 & 1.13 & $\mathrm{~mol}_{\mathrm{O} 2} \mu \mathrm{mol}_{h v}$ & \\
\hline${\overline{\phi^{\prime}}}_{O_{2}}$ & $1.1 \times 10^{-7}$ & $1.1 \times 10^{-7}$ & $\mathrm{~mol}_{\mathrm{O} 2} / \mu \mathrm{mol}_{h v}$ & \\
\hline$M_{X}$ & 0.024 & 0.024 & $\mathrm{~kg}_{X} \mathrm{C}-\mathrm{mol}^{-1}$ & \\
\hline$v_{\mathrm{NADH}_{2}-\mathrm{O}_{2}}$ & 2 & 2 & - & \\
\hline$K$ & 40,000 & 30,000 & $\mu \mathrm{mol}_{\mathrm{h} v} / \mathrm{kg} / \mathrm{s}$ & Deduced from experiments \\
\hline$K_{r}$ & 500 & 150 & $\mu \mathrm{mol}_{\mathrm{h} v} / \mathrm{kg} / \mathrm{s}$ & \\
\hline $\mathcal{A}_{c}$ & 2,800 & 1,500 & $\mu \mathrm{mol}_{\mathrm{h} v} / \mathrm{kg} / \mathrm{s}$ & \\
\hline
\end{tabular}

computation time in PBR modeling, but needs to be combined with a specific determination of kinetic growth model parameters for better accuracy.

\section{Investigation of the effects of incident angle and light spectrum in a thin flat-panel PBR}

Theoretical Preliminary Analysis. As our model was able to take light spectrum and light incident angle into individual consideration, it was used for a preliminary theoretical investigation of the influence of these parameters on PBR biomass productivity. It was then applied to the thin-flat panel PBR working with normal $\left(\theta=0^{\circ}\right)$ and oblique $\left(\theta=60^{\circ}\right)$ incident angles, and with white and red light.

We first assumed a constant total pigment content of $5 \%$ and a constant dry weight biomass concentration of $2 \mathrm{~g} / \mathrm{L}$ (values deduced from model predictions for operating conditions applied on thin-flat panel PBRs as described in the next section, i.e., constant PFD of $100 \mu \mathrm{mol}_{\mathrm{h} v} / \mathrm{m}^{2} / \mathrm{s}$ and constant dilution rate of $0.015 \mathrm{~h}^{-1}$ ). Based on these values, the evolution down the culture depth of local values of specific rate of photon absorption $\mathcal{A}$, and specific rate of oxygen production $J_{\mathrm{O}_{2}}$ were predicted (Eq. 6, and Eq. 5 combined with 6). Corresponding PBR biomass productivity $P_{x}$ was then deduced (Eqs. 1 and 9).

Profiles of the local rates of photon absorption $\mathcal{A}$ and of oxygen production $J_{\mathrm{O}_{2}}$ are presented in Figure 5. For normal incidence, closely similar evolutions were obtained for the two light spectra, resulting in similar light extinction and growth rates, with a slight advantage for red light. It is interesting to compare the evolution of the spectrum of the local rate of photon absorption. The results are represented in Figure 6 for white (Figure 6a) and red (Figure 6b) light. Although the attenuation profiles of spectrally averaged values were found to be similar (Figure 5), we obtained very different evolutions in their corresponding spectra. For red light, because of its narrow emission range (i.e., 600$700 \mathrm{~nm}$ ), the spectrum of the local rate of photon absorption remained almost unchanged throughout the depth of the PBR. However, for the white light, because of its emission over the entire PAR range (i.e., 400-700 nm) and of the non-homogeneous spectral absorption by the culture (as represented by the spectral distribution of the mass absorption coefficient $E_{a \lambda}$ ), a significant evolution of the spectrum of photon absorption rate was observed with the depth of culture. Because of a higher absorption, red and blue radiation wavelengths were rapidly absorbed (chlorophyll absorption peaks). The wavelength range remaining deep in the culture was, thus, mainly green light. Although cell pigmentation favored the absorption of these wavelengths less, they were still absorbed. The deeper penetration of less well absorbed wavelengths thus favorably impacted the local values of pho- ton absorption rate, and so because cells still received light energy for their growth, values of $J_{\mathrm{O}_{2}}$ deep in the culture were found to be higher for white light. White light, thus, had a positive effect by decreasing the influence of dark volume through allowing the less absorbed wavelengths to penetrate deeper into the culture bulk.

Theoretical predictions for an oblique incident angle are given in Figure 5. As already observed in Pruvost et al., ${ }^{26}$ non-normal incident angle leads to a greater decrease in available light with culture depth due to an increased light attenuation distance of collimated light on increasing the incident angle (Figure 2b). The same behavior was observed here. Even if the same PFD was applied to the PBR surface (i.e., same energy received on the PBR surface in all cases), the rate of photons absorption decreased more rapidly. This resulted in a lower extinction depth of light energy (illuminated fractions of $\gamma=0.52$ and $\gamma=0.48$ for white and red light, respectively). Surprisingly, light attenuation, as represented by $\mathcal{A}$, appeared more accentuated in the case of the red light spectrum, with a marked decrease, especially in the second half of the culture depth. This directly influences local values of $J_{\mathrm{O}_{2}}$ whose evolution differed depending on illumination conditions. Biomass concentration and PFD being the same in all the simulations, this marked influence of the light spectrum was again directly explained by the spectrally dependent light absorption of microalgae. Like at normal incidence, the deeper penetration of less wellabsorbed wavelengths (range of green radiation) impacted favorably on the local values of photon absorption rates (and consequently on $J_{\mathrm{O}_{2}}$ ) within the culture depth. In the conditions of high incident angle, for the first half of the culture depth, local values of $J_{\mathrm{O}_{2}}$ were similar, but then split, with a difference that widened with increasing culture depth. Under red radiation, the sharp decrease in local rate of photon absorption resulted in a fast decrease in local values of oxygen production due to less available light energy in the culture depth. We note that although light extinction depth was very close in both cases (illuminated fractions of $\gamma=0.62$ and $\gamma=0.69$ under normal incidence for white and red radiations, respectively; $\gamma=0.52$ and $\gamma=0.48$ under oblique incidence for white and red radiations, respectively), the negative effect of the dark zone was found to be more marked under the red spectrum because of the stronger decrease in local rate of photon absorption, with accordingly a greater decrease in $J_{O_{2}}$ values than under white light. As a consequence, local values of $J_{O_{2}}$ deep in culture were very low for oblique red illumination conditions.

The spatial integration of the local values of $J_{O_{2}}$ lets us calculate the resulting biomass productivity for corresponding operating conditions (Eqs. 1 and 9). Results are given in Figure 5 and in Table 2. We confirm the marked influence of red radiation and especially oblique incident angle on 
a
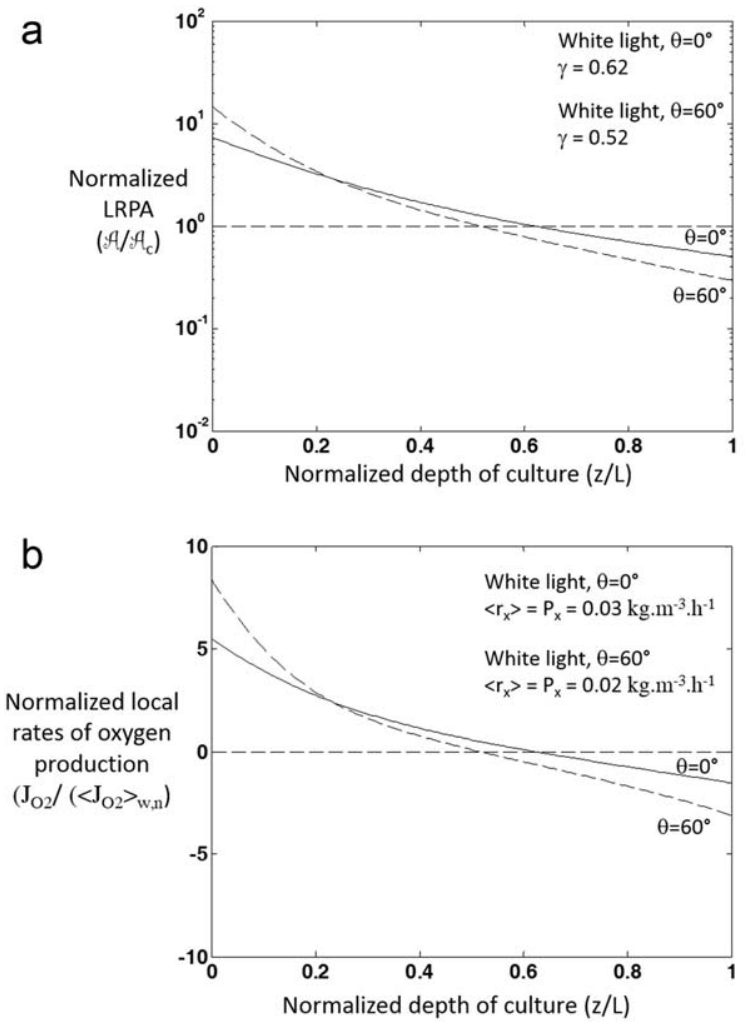
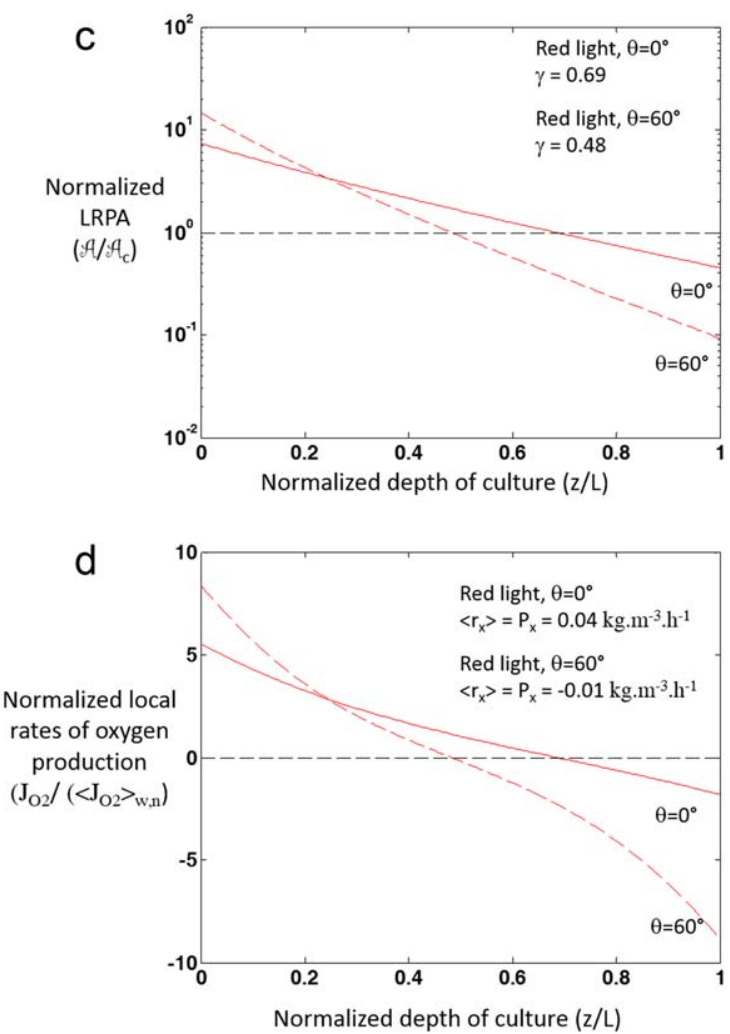

Figure 5. Theoretical evolution of the local rate of photon absorption $\mathcal{A}$ (a and c) and of the local specific rate of oxygen production $J_{O_{2}}$ (b and d) along the PBR depth, assuming constant biomass $\left(2 \mathrm{~kg} \cdot \mathrm{m}^{-3}\right)$ and pigment content $(5 \%)$ for white (a and b) and red (c and d) emission light spectra (thin-flat panel PBR, $q_{0}=100 \mu \mathrm{mol}_{\mathrm{hn}} \cdot \mathrm{m}^{2} \cdot \mathrm{s}^{-1}, D=0.0015 \mathrm{~h}^{-1}$ ). For convenience, values were normalized with the rate of photon absorption at compensation point $\left(\mathcal{A}_{c}\right)$ for LRPA and with the averaged specific rate of energy production $\left\langle J_{O_{2}}\right\rangle$ achieved for white light and normal incidence $\left(\left\langle J_{O_{2}}\right\rangle_{w n}=0.78 \mathbf{m o l}_{\mathrm{O}_{2}} \cdot \mathbf{k g}_{x} \cdot \mathbf{h}^{-1}\right.$, equivalent to $\left.P_{x w, n}=\left\langle r_{x}\right\rangle_{w, n}=0.03 \mathrm{~kg} \cdot \mathrm{m}^{-3} \cdot \mathbf{h}^{-1}\right)$. For all, solid and dashed lines are for normal and $60^{\circ}$ incident angle, respectively.

light extinction profiles and resulting biomass productivity, even in the same conditions of PFD and biomass characteristics affecting light transfer (i.e., biomass concentration and radiative properties). For the extreme case of red light and oblique incidence, no growth is theoretically allowed (the model predicts negative growth rates). In the next section we compare this preliminary theoretical conclusion with actual experimental results.

Experimental Results Obtained in a Thin-Flat Panel $P B R$. PBR biomass productivity was experimentally determined for the two incident spectra (white, red) and incident angles $\left(0^{\circ}, 60^{\circ}\right)$ investigated in the previous section. The thin flat-panel PBR was operated in continuous mode with constant dilution rate. For each light spectrum and incident angle, the PFD was adjusted to $100 \mu \mathrm{mol}_{\mathrm{h} v} / \mathrm{m}^{2} / \mathrm{s}$; the light source emission power was adjusted to correct for the decrease in light received on the PBR surface due to the higher incident angle (see PBR description in section "Cultivation systems and lighting"). The experimental results are presented in Table 2 (see also Figure 7). Comparing with results obtained with the torus $\mathrm{PBR}$, we can note the positive effect of increasing PBR specific illuminated surface (i.e., reducing PBR depth, $a_{\text {light }}=1 / L$ ) in the intensification of biomass productivities and concentration, with a five- to eightfold increase in these values in the thin-flat panel PBR (here obtained for a halved PFD value).

Both light spectrum and incident angle were found to influence resulting biomass productivity, and especially the incident angle, which proved to have the more marked effect, as already found theoretically in the previous section. This non-negligible effect of the incident angle on the biomass productivity confirms the conclusion of Pruvost et al. ${ }^{26}$ where non-normal incidence was found to influence significantly the biomass productivity in solar PBR, and so needs to be taken into account in solar PBR modeling.

Interestingly, even if an effect of both light spectrum and incident angle was experimentally observed, it was found to be less marked than expected from the previous theoretical treatment. Significant biomass production was observed experimentally in all cases, even in the more drastic conditions of red light and oblique incidence. Experimentally, the normal incidence still corresponded to maximal biomass productivity, but non-normal incidence caused only a modest decrease in productivity $(<25 \%)$. Surprisingly, although a small change in biomass concentration was found, a more marked change was found in the pigment content, which was strongly affected by illumination conditions (from $4.4 \%$ to $7.7 \%$ ). Increasing the incident angle led to a higher pigment content, especially for white light $(5 \%-7.7 \%)$. For red light, a lower pigment content was observed $(4.4 \%$ and $5.2 \%$ for normal and $60^{\circ}$ incident angle, respectively). The effects of these significant changes in pigment contents are described in detail in the following section.

Model Prediction and In-Depth Analysis of the Influence of Incident Angle and Light Spectrum. To investigate the influence of the pigment acclimation, the model was used to predict PBR biomass productivities and concentrations by introducing values of pigment content obtained experimentally 

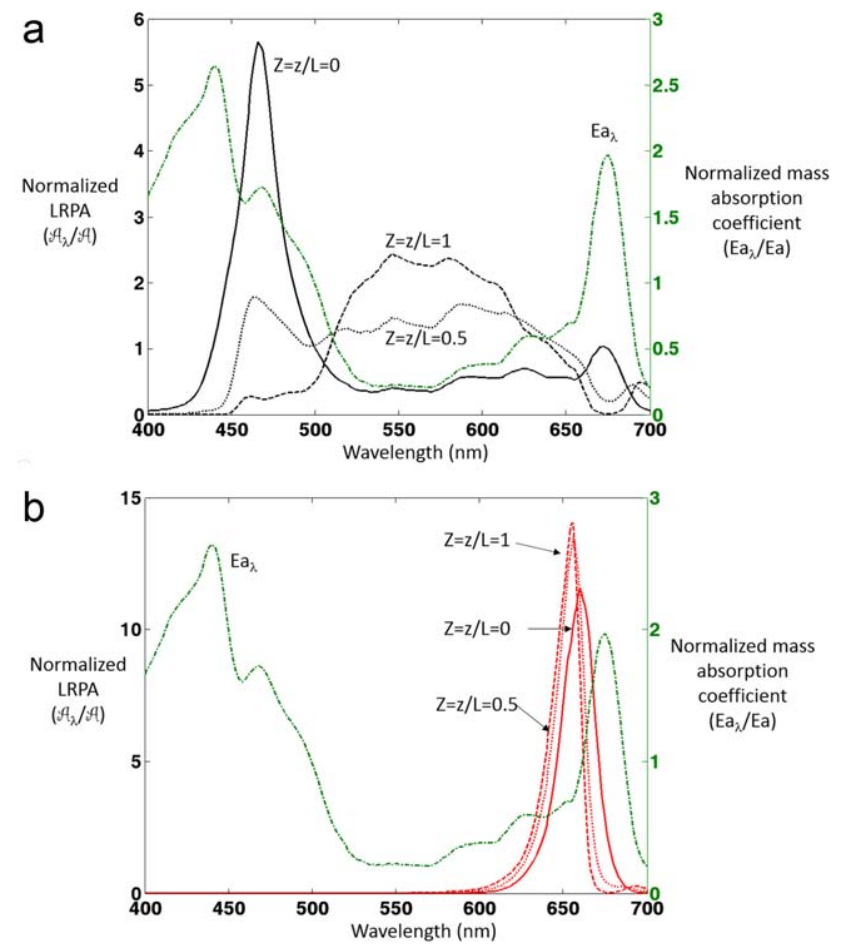

Figure 6. Evolution of the spectral local rate of photon absorption deep in the culture for white (a) and red (b) light emission spectra. The spectral distribution of the mass absorption coefficient of the culture was added (dashdot line, corresponding averaged value $E_{a}=238 \mathrm{~m}^{2} / \mathrm{kg}$ ). Calculations were made for normal incidence, constant biomass $\left(2 \mathrm{~kg} / \mathrm{m}^{3}\right)$ and pigment content $(5 \%)$. Values are given for $z / L=0$ (solid line), $z / L=0.5$ (dotted line), and $z / L=1$ (dashed line), and were normalized by their corresponding spectrally averaged values (see Eq. 5 for local rate of photon absorption).

for each case. Surprisingly, and unlike predictions with a constant pigment content of 5\% (Figure 5), the predictions were found to be very close to the real ones, with an averaged relative error of around $12 \%$ (see Table 2), confirming the major role of pigment acclimation on PBR performance.

Corresponding model predictions are given in Figure 7 for a full range of dilution rate values. This confirms that experimental values were not obtained in the optimal operating conditions leading to maximal productivities, but for conditions leading to high biomass concentration and so high light attenuating conditions. As already observed for the torus PBR, a reduction in the model's prediction accuracy was found for these conditions, which could also certainly apply to the thinflat panel PBR. Given also that the case under study, a thinflat panel PBR, is not easy to investigate experimentally in practice because of the very specific conditions of illumination, we can consider that our model performed fairly well. We must remember that the same model parameters as obtained on the torus PBR were applied. The model was thus able to represent the significant decrease in PBR depth (i.e., from 0.04 to $0.0076 \mathrm{~m}$ ) leading to a biomass concentration and productivity increase, and also the change in PFD characteristics (intensity, light spectrum, and incident angle).

Model predictions confirmed the complex effect of both light spectra and incident angle on the PBR biomass productivity. For conditions of full light absorption (i.e., $D<D_{\text {opt }}$ ), oblique incidence had a negative impact on biomass produc- tivity, although the same PFD was applied on PBR optical surface (i.e., same light energy received). This is already discussed in Pruvost et al. ${ }^{9}$ for solar fixed PBR working with non-normal direct radiation, and was explained by the higher decrease in light penetration, which was thus less favorable for photosynthetic conversion. However, such an effect decreases with the decreasing light absorption as observed here with the increase in dilution rate leading to lower biomass concentration. For higher dilution rates leading to light transmission $\left(D>D_{o p t}\right)$, the opposite result was obtained, and higher biomass productivities were therefore achieved. Decreased light penetration due to incident angle increase was found in this particular case to be beneficial, by decreasing the negative effect of light transmission for low biomass concentration. We note that the same tendency was observed for both light spectra. White light exhibited a less marked influence of the incident angle. As already discussed, this is explained by the spectral light attenuation, with emission wavelengths less well absorbed but going deeper into the culture. This mitigates the effect of light attenuation and thereby the effect of high incidence angle.

To conclude this study, the model was lastly used for a detailed investigation of the effect of pigment change on the modification of light attenuation conditions inside the PBR bulk and how it in turn affects the photosynthetic conversion (as represented by the local values of growth rates $J_{O_{2}}$ ). The results are presented in Figure 8. Local values of rates of photon absorption and photosynthetic conversion were found to be greatly affected by the pigment evolution. In a general manner, this tended surprisingly to attenuate the negative effects of high light attenuation conditions as obtained with red light spectrum and oblique incident angle. Compared with predictions assuming constant pigment content (Figure 5), higher rates of photon absorption were found in the bottom part of the PBR, with correspondingly higher local growth rates. We note that this increase was not explained only by a decrease in pigment content, as oblique incidence was found to increase the pigment content, contrary to red light, which led to lower pigment content than with white light. This is explained by the complex relationship between the specific rate of photon absorption and the parameters affecting this value, such as the pigment content and biomass concentration. The specific rate of photon absorption is related to the mass absorption coefficient $E_{a}$, which increases with pigment content, and with the available irradiance $G$, which decreases with the increase in pigment content and biomass concentration. As a result, the effect of the modification of one parameter such as the pigment content on the resulting light energy absorption is difficult to predict without accurate modeling. This emphasizes the relevance of applying a rigorous treatment of the radiative transfer part in the prediction of photosynthetic growth in a PBR. As already observed by Kandilian et al. ${ }^{16}$ but for lipid production, the specific rate of photon absorption was found to be meaningful in the very different case of biomass production under various light spectra and incident angles. This confirms the relevance of this physical quantity in the modeling and understanding of microalgal growth in PBR technology.

\section{Discussion of the effect of incident angle and light spectrum on $P B R$ productivity}

Our findings confirm that both light spectrum and incident angle influence PBR productivity, by affecting light attenuation conditions in the culture bulk, and thereby the resulting 
Table 2. Experimental Results of $C$. vulgaris Culture in a Thin-Flat Panel PBR for White and Red Light and With Normal and Oblique Incidence, and Comparison with Theoretical Predictions of the PBR Model (See Text for Details)

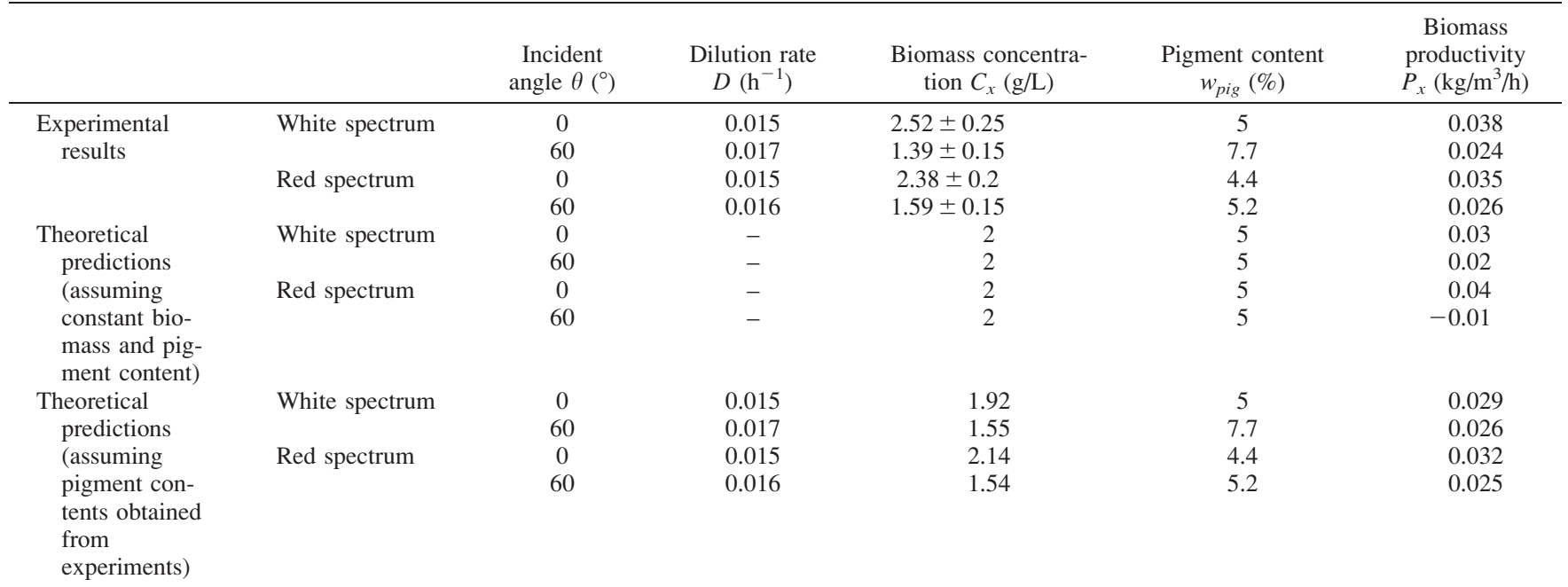
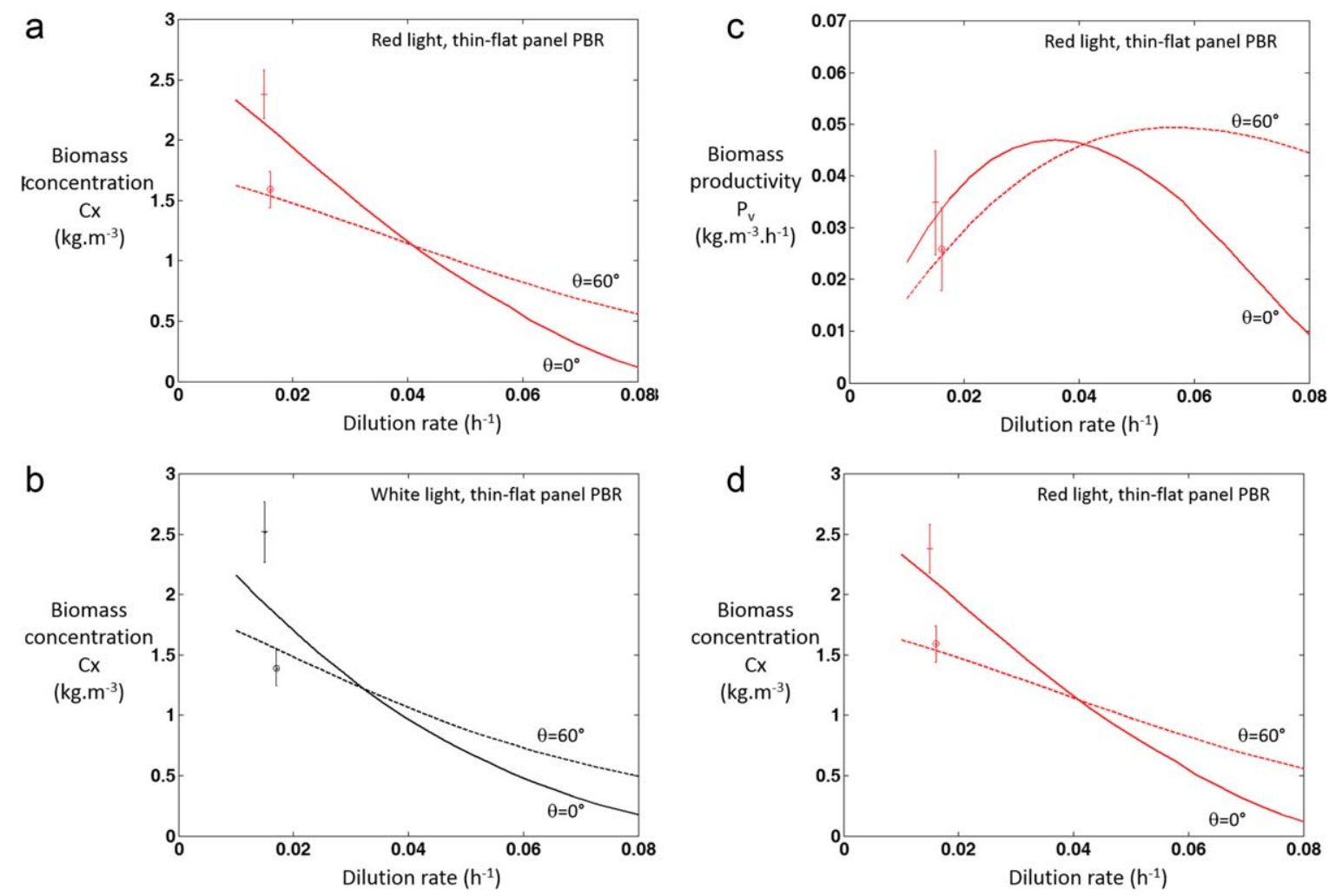

Figure 7. Volumetric biomass productivity (a and c), biomass concentration (b and d) as a function of the dilution rate for $C$. vulgaris culture in a thin flat-panel PBR for white (a and b) and red (c and d) light emission spectra (plus and circle marks are experimental data for normal and oblique incidence, respectively; solid and dashed lines are theoretical predictions for normal and oblique incidence, respectively).

photosynthetic growth rate and biomass productivity. This is not surprising since any modification of the light attenuation field can be assumed to modify the photosynthetic conversion. Our study emphasizes, however, that pigment acclimation plays a major role, influencing rates of photon absorption and then photosynthetic growth, and reducing in all cases the negative influence of dark volumes on resulting PBR productivity. With pigment acclimation, kinetic performance of the PBR was only moderately reduced (around $30 \%$ decrease, against 50\%-100\% without pigment acclimation). The exact underlying biological mechanism is not clear, and lies outside the scope of this study. But this important observation clearly indicates that pigment acclimation has to be taken into account, as it greatly influences the cultivation process. In our study devoted to biomass production in PBR, it was found to act positively.

Our results can also be discussed in terms of PBR optimization. For example, the choice of a light spectrum promoting wavelengths absorbed by cell pigments (i.e., red light) is often put forward as of interest in PBR engineering. Our results indicate, however, that this is not always the case, and it depends on the light absorption conditions in the 

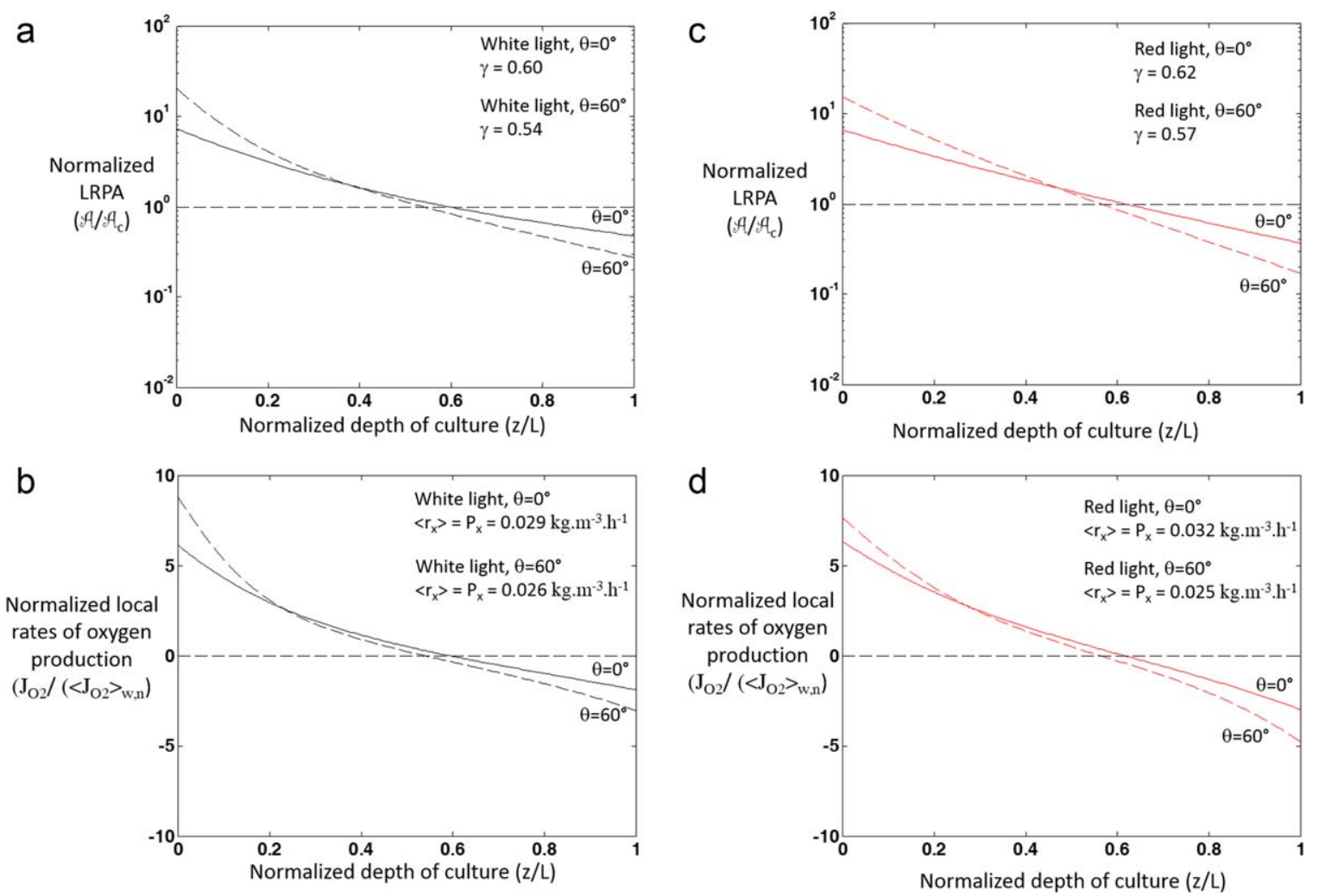

Figure 8. Theoretical evolution of the local rate of photon absorption $\mathcal{A}$ (a and c) and of the local specific rate of oxygen production $\mathrm{J}_{\mathrm{O}_{2}}$ (b and d) along the PBR depth, as predicted by the model when considering pigment content obtained experimentally at steady-state in a thin-flat panel PBR. Results are given for white (a and b) and red (c and d) emission light spectra. For convenience, values were normalized with the rate of photon absorption at compensation point $\left(\mathcal{A}_{c}\right)$ for LRPA and with the averaged specific rate of energy production $\left\langle J_{O_{2}}\right\rangle$ achieved for white light and normal incidence $\left(\left\langle J_{O_{2}}\right\rangle_{w, n}=\right.$ $0.78 \mathrm{~mol}_{\mathrm{O}_{2}} / \mathrm{kg}_{x} / \mathrm{h}$, equivalent to $\left.\boldsymbol{P}_{x, w}=\left\langle\boldsymbol{r}_{x}\right\rangle_{w, n}=0.03 \mathrm{~kg} / \mathrm{m}^{3} / \mathrm{h}\right)$. For all, solid and dashed lines are for normal and $60^{\circ}$ incident angle, respectively.

culture bulk. For high light absorption conditions typical of PBR operation (i.e., high biomass concentration), a white light spectrum leads to a higher biomass productivity due to a deeper light penetration because of the presence of less well-absorbed wavelengths in the light emission (i.e., green part of the white light spectrum). On the contrary, for moderate absorption conditions (i.e., near luminostat regime), biomass productivity was found to be slightly increased in red light. Less energy was lost by the transmission of poorly absorbed wavelengths. But the increase in productivity was found to be very low (around 10\%) in this case. Hence, the utility of an optimized light spectrum such as red light for PBR intensification is evidently moderate. The main benefit of using red light here is in terms of light attenuation control. Through a narrower bandwidth of wavelengths in radiation emission, red light enables easier control of the light attenuation field. The light spectrum was found to be largely unchanged throughout the culture depth, which was not the case for white light (progressive variation toward green light). This could be of interest for fundamental research purposes, to make a close investigation of the relationship between light attenuation field and photosynthetic conversion in light-attenuating conditions of PBR.

\section{Conclusion}

The effect of different illumination conditions, namely light spectrum and incident angle, on $C$. vulgaris growth in
PBR was investigated. A model able to take into account these different conditions is proposed, and was validated on both torus PBR and thin layer flat-panel PBR, the latter permitting high-cell density culture.

Both red light spectrum and oblique incident angles were found to affect biomass productivity in a PBR by modifying rates of photon absorption along the PBR depth. The influence of red light spectrum was found to depend on light attenuation conditions, with a positive effect only in low light attenuating conditions. White light was found to be more beneficial in high attenuating conditions, because of a deeper penetration of low absorbed wavelength such as in the green part of the light spectrum. In the case of full light absorption, oblique incident angle was found to have a negative influence on biomass productivity whatever the incident light spectrum, showing the importance of allowing for it when, for example, modeling on solar PBRs where nonnormal incidence prevails owing to the sun's relative travel.

For all the conditions investigated, we found a high pigment adaptation of $C$. vulgaris to illumination conditions (i.e., incident angle, light spectrum). Using the model, the effect of such pigment adaption was systematically investigated. We observed that, quite surprisingly, in all cases the pigment acclimation greatly attenuated the negative impact of non-optimized illumination conditions. For both red radiation and high incident angle, pigment acclimation led to deeper light penetration in the culture, resulting in a higher local growth rate deep in the culture. This tended to sustain 
kinetic performance of the process (i.e., biomass productivity).

In conclusion, this study confirms the general utility of accurately relating the light transfer conditions (and more specially the distribution of specific rate of light absorption $\mathcal{A})$ to the growth kinetics. Relevant features affecting PBR productivity such as oblique incident angle or various light spectra can then be predicted with accuracy.

Our findings open up several perspectives. Regarding the modeling approach, a slight discrepancy in the model prediction was observed for high light attenuation conditions. This can certainly be attributed to the formulation of the kinetic growth model, and more precisely to the formulation of the respiration term activity whose influence prevails in high light attenuation conditions. This needs to be improved for a better representation of all the conditions encountered in the PBR operation.

In all the cases investigated, photoacclimation was found to be highly relevant. This should also be investigated more thoroughly. In our study, experimental pigment content values obtained experimentally were introduced in the model. A predictive determination of the pigment content depending on the operating conditions should be of interest to extend the applicability of the model.

This work was based only on steady-state observations made in continuous mode and with constant irradiation conditions. It would be interesting to investigate in more depth the dynamic acclimation of pigments in conditions representative of solar use, where dynamic variations of light intensity, incident angle and light spectrum occur.

\section{Acknowledgments}

This work was supported by the French regional research program for energy (PERLE2) and by the French National Research Agency project DIESALG (ANR-12-BIME-0001-02).

\section{Notation}

$\mathcal{A}=$ local specific rate of photon absorption, $\mu \mathrm{mol}_{\mathrm{h} v} / \mathrm{kg} / \mathrm{s}$

$\mathcal{A}_{c}=$ specific rate of photon absorption at compensation point, $\mu \mathrm{mol}_{\mathrm{h} v} / \mathrm{kg} / \mathrm{s}$

$a_{\text {light }}=$ specific illuminated area for the photobioreactor, $\mathrm{m}^{-1}$

$b=$ back-scattered fraction for radiation, dimensionless

$C_{X}=$ biomass concentration, $\mathrm{kg} / \mathrm{m}^{3}$

$D=$ dilution rate $\mathrm{h}^{-1}$ or s ${ }^{-1}$

$E_{a}=$ mass absorption coefficient, $\mathrm{m}^{2} / \mathrm{kg}$

$E_{s}=$ mass scattering coefficient, $\mathrm{m}^{2} / \mathrm{kg}$

$G=$ local spherical irradiance, $\mu \mathrm{mol}_{\mathrm{h} v} / \mathrm{m}^{2} / \mathrm{s}$

$J_{O_{2}}=$ local specific rate of oxygen production or consumption, $\mathrm{mol}_{\mathrm{O}_{2}} \mathrm{~kg}_{x}^{-1} \mathrm{~s}^{-1}$

$J_{\mathrm{NADH}_{2}}=$ specific rate of cofactor regeneration on the respiratory chain, mol $_{\mathrm{NADH}_{2}} \mathrm{~kg}_{x}^{-1} \mathrm{~s}^{-1}$

$K=$ half saturation constant for photosynthesis, $\mu \mathrm{mol}_{\mathrm{h} v} / \mathrm{kg} / \mathrm{s}$

$K_{r}=$ half saturation constant describing the inhibition of respiration in light, $\mu \mathrm{mol}_{\mathrm{h} v} / \mathrm{kg} / \mathrm{s}$

$L=$ depth of the rectangular photobioreactor, $\mathrm{m}$

$P_{X}=$ biomass volumetric productivity, $\mathrm{kg} / \mathrm{m}^{3} / \mathrm{s}$ or $\mathrm{kg} / \mathrm{m}^{3} / \mathrm{h}$

$q=$ total radiation received on photobioreactor surface (same as photon flux density PFD), $\mu \mathrm{mol}_{\mathrm{h} v} / \mathrm{m}^{2} / \mathrm{s}$

$r_{x}=$ volumetric biomass production rate, $\mathrm{kg} / \mathrm{m}^{3} / \mathrm{s}$ or $\mathrm{kg} / \mathrm{m}^{3} /$ $\mathrm{h}$

$\mathrm{R}_{\mathrm{S}}=$ Rear side reflective index, dimensionless

$S_{\mathrm{L}}=$ illuminated surface of the photobioreactor, $\mathrm{m}^{2}$
$V_{r}=$ photobioreactor volume, $\mathrm{m}^{3}$

$z=$ depth of culture, $\mathrm{m}$

\section{Greek letters}

$\alpha=$ linear scattering modulus, dimensionless

$\gamma=$ fraction for working illuminated volume in the photobioreactor, dimensionless

$\delta=$ extinction coefficient for the two-flux method, $\mathrm{m}^{-1}$

$\lambda=$ light wavelength, $\mathrm{m}$

$v_{\mathrm{NADH}_{2}-\mathrm{O}_{2}}=$ stoichiometric coefficient of cofactor regeneration on the respiratory chain, dimensionless

$v_{O_{2}-X}=$ stoichiometric coefficient of the oxygen production, dimensionless

$\theta=$ incident angle (defined from the outward normal of the PBR), rad

$\rho_{M}=$ maximum energy yield for photon conversion, dimensionless

$\tau_{p}=$ hydraulic residence time, $\mathrm{h}$

$\overline{\phi_{O_{2}}^{\prime}}=$ mean mass quantum yield for the Z-scheme of photosynthesis, $\mathrm{mol}_{\mathrm{O}_{2}} / \mathrm{mmol}_{\mathrm{hn}}$

\section{Subscripts}

dark $=$ related to a dark zone in the photobioreactor

light $=$ related to an illuminated zone in the photobioreactor

$o p t=$ related to the optimal value for residence time

\section{Literature Cited}

1. Spolaore P, Joannis-Cassan C, Duran E, Isambert A. Commercial applications of microalgae. J Biosci Bioeng. 2006;101: 87-96.

2. Pruvost J, Cornet JF. Knowledge models for engineering and optimization of photobioreactors. In Posten P, Walter C, editor. Microalgal Biotechnology. Berlin: De Gruyter GmbH \& Co. KG; 2012:181-224.

3. Wallen DG, Geen GH. Light quality in relation to growth, photosynthetic rates and carbon metabolism in two species of marine plankton algae. Marine Biol. 1971;10:34-43.

4. Figueroaa FL, Aguileraa A, Niella FX. Red and blue light regulation of growth and photosynthetic metabolism in Porphyra umbilicalis (Bangiales, Rhodophyta). Eur J Phycol. 1995;30: 11-18.

5. Wang $\mathrm{CY}, \mathrm{Fu} \mathrm{CC}$, Liu YC. Effects of using light-emitting diodes on the cultivation of Spirulina platensis. Biochem Eng J. 2007;37:21-25.

6. Zhao Y, Wang J, Zhang H, Yan C, Zhang Y. Effects of various LED light wavelengths and intensities on microalgae-based simultaneous biogas upgrading and digestate nutrient reduction process. Bioresour Technol. 2013;136:461-438.

7. Farges B, Laroche C, Cornet JF, Dussap CG. Spectral kinetic modeling and long-term behavior assessment of Arthrospira platensis growth in photobioreactor under red $(620 \mathrm{~nm})$ light illumination. Biotechnol Prog. 2009;25:151-162.

8. Hess JL, Tolbert NE. Changes in chlorophyll a/b ratio and products of $14 \mathrm{co} 2$ fixation by algae grown in blue or red light. Plant Physiol. 1967;42:1123-1130.

9. Pruvost J, Cornet JF, Goetz V, Legrand J. Modeling dynamic functioning of rectangular photobioreactors in solar conditions. AIChE J. 2011;57:1947-1960.

10. Takache H, Pruvost J, Cornet JF. Kinetic modeling of the photosynthetic growth of Chlamydomonas reinhardtii in a photobioreactor. Biotechnol Prog. 2012;28:681-692.

11. Pruvost J, Van Vooren G, Le Gouic B, Couzinet-Mossion A, Legrand J. Systematic investigation of biomass and lipid productivity by microalgae in photobioreactors for biodiesel application. Bioresour Technol. 2011;102:150-158.

12. Pottier L, Pruvost J, Deremetz J, Cornet JF, Legrand J, Dussap CG. A fully predictive model for one-dimensional light attenuation by Chlamydomonas reinhardtii in a torus reactor. Biotechnol Bioeng. 2005;91:569-582. 
13. Pruvost J, Pottier L, Legrand J. Numerical investigation of hydrodynamic and mixing conditions in a torus photobioreactor. Chem Eng Sci. 2006;61:4476-4489.

14. Ritchie RJ. Consistent sets of spectrophotometric chlorophyll equations for acetone, methanol and ethanol solvents. Photosynth Res. 2006;89:27-41.

15. Strickland JDH, Parsons TR. A Practical Handbook of Seawater Analysis: Pigment Analysis. Ottawa: Bulletin of Fisheries Research Board of Canada; 1968:167.

16. Kandilian R, Pruvost J, Legrand J, Pilon L. Influence of light absorption rate by Nannochloropsis oculata on triglyceride production during nitrogen starvation. Bioresour Technol. 2014; 163:308-319.

17. Irazoqui HA, Cerdá J, Cassano AE. The radiation field for the point and line source approximations and the three-dimensional source models. Applications to photoreactions. Chem Eng J. 1976;11:27-37.

18. Spadoni G, Bandini E, Santarelli F. Scattering effects in photosensitized reactions. Chem Eng Sci. 1978;33:517-524.

19. Dauchet, J. Analyse radiative des photobioreacteurs. PhD Thesis, Universite Blaise Pascal - Clermont-Ferrand, France ( ${ }^{\circ}$ ordre 2304 - in french); 2012.

20. Cogne G, Rugen M, Bockmayr A, Titica M, Dussap CG, Cornet JF, Legrand J. A model-based method for investigating bioenergetic processes in autotrophically growing eukaryotic microalgae: application to the green alga Chlamydomonas reinhardti. Biotechnol Prog. 2011;27:631-640.

21. Cournac L, Musa F, Bernard L, Guedeney G, Vignais P, Peltier G. Limiting steps of hydrogen production in Chlamydomonas reinhardtii and Synechocystis PCC 6803 as analysed by lightinduced gas exchange transients. Int J Hydrogen Energy. 2002; 27:1229-1237.

22. Peltier G, Thibault P. Uptake in the Light in Chlamydomonas. Evidence for persistent mitochondrial respiration. Plant Physiol. 1985;79:225-230.

23. Cornet JF, Dussap CG, Gros JB. A simplified monodimensional approach for modeling coupling between radiant light transfer and growth kinetics in photobioreactors. Chem Eng Sci. 1995; 50:1489-1500.

24. Cornet JF, Dussap CG, Gros JB. Kinetics and energetics of photosynthetic micro-organisms in photobioreactors: application to Spirulina growth. Adv Biochem Eng Biotechnol. 1998;59:155-224.

25. Lee E, Pruvost J, He X, Munipalli R, Pilon L. Design tool and guidelines for outdoor photobioreactors. Chem Eng Sci. 2014; 106:18-29.

26. Pruvost J, Cornet JF, Goetz V, Legrand J. Theoretical investigation of biomass productivities achievable in solar rectangular photobioreactors for the cyanobacterium Arthrospira platensis. Biotechnol Prog. 2012;28:699-714.

27. Kandilian R, Lee E, Pilon L. Radiation and optical properties of Nannochloropsis oculata grown under different irradiances and spectra. Bioresour Technol. 2013;137:63-73.

28. Bohren CF, Huffman DR. Absorption and Scattering of Light by Small Particles. New York: Wiley; 1983.

29. Dauchet J, Blanco S, Cornet JF, Fournier R. Calculation of the radiative properties of photosynthetic microorganisms. J Quant Spectrosc Radiat Transf. 2015;161:60-84.
30. Soulies A, Pruvost J, Legrand J, Castelain C, Burghelea TI. Rheological properties of suspensions of the green microalga chlorella vulgaris at various volume fractions. Rheol Acta. 2013; 52:589-605.

31. Cornet JF. Calculation of optimal design and ideal productivities of volumetrically lightened photobioreactors using the constructal approach. Chem Eng Sci. 2010;65:985-998.

32. Takache H, Christophe G, Cornet JF, Pruvost J. Experimental and theoretical assessment of maximum productivities for the microalgae Chlamydomonas reinhardtii in two different geometries of photobioreactors. Biotechnol Prog. 2010;26:431-440.

33. Carvalho AP, Silva SO, Baptista JM, Malcata FX. Light requirements in microalgal photobioreactors: an overview of biophotonic aspects. Appl Microbiol Biotechnol. 2011;89:1275-1288.

34. Grima ME, Fernandez AFG, Camacho GF, Chisti Y. Photobioreactors: light regime, mass transfer, and scaleup. J Biotechnol. 1999;70:231-247.

35. Richmond A. Principles for attaining maximal microalgal productivity in photobioreactors: an overview. Hydrobiologia. 2004;512:33-37.

36. Teo CL, Atta M, Bukhari A, Taisir M, Yusuf AM, Idris A. Enhancing growth and lipid production of marine microalgae for biodiesel production via the use of different LED wavelengths. Bioresour Technol. 2014;162:38-44.

37. Das P, Lei W, Aziz S, Obbard JP. Enhanced algae growth in both phototrophic and mixotrophic culture under blue light. Bioresour Technol. 2011;102:3883-3887.

38. Baba M, Kikuta F, Suzuki I, Watanabe MM, Shiraiwa Y. Wavelength specificity of growth, photosynthesis, and hydrocarbon production in the oil-producing green alga Botryococcus braunii. Bioresour Technol. 2012;109:266-270.

39. Kim TH, Lee Y, Han SH, Hwang SJ. The effects of wavelength and wavelength mixing ratios on microalgae growth and nitrogen, phosphorus removal using Scenedesmus sp. for wastewater treatment. Bioresour Technol. 2013;130:75-80.

40. Richmond A. Handbook of Microalgal Culture: Biotechnology and Applied Phycology. Oxford, UK: Blackwell Sciences Ltd; 2004.

41. Josrgensen EG. The adaptation of plankton algae IV. Light adaptation in different algal species. Physiol Plant. 1969;22: $1307-1315$

42. Falkowski PG, LaRoche J. Minireview: acclimation to spectral irradiance in algae. J Phycol. 1991;27:8-14.

43. Van Vooren G, Le Grand F, Legrand J, Cuine S, Peltier G, Pruvost J. Investigation of fatty acids accumulation in Nannochloropsis oculata for biodiesel application. Bioresour Technol. 2012; 124:421-432.

44. Roels JA. Energetics and Kinetics in Biotechnology. Amsterdam: Elsevier Biomedical Press; 1983.

45. Cornet JF, Dussap CG. A simple and reliable formula for assessment of maximum volumetric productivities in photobioreactors. Biotechnol Prog. 2009;25:424-435. 